\title{
CONSTRUCTING COMMON FACTORS FROM CONTINUOUS AND CATEGORICAL DATA
}

\author{
Serena $\mathrm{Ng}$ \\ Department of Economics \\ Columbia University*
}

August 2012

\begin{abstract}
The method of principal components is widely used to estimate common factors in large panels of continuous data. This paper first reviews alternative methods that obtain the common factors by solving a Procrustes problem. While these matrix decomposition methods do not specify the probabilistic structure of the data and hence do not permit statistical evaluations of the estimates, they can be extended to analyze categorical data. This involves the additional step of quantifying the ordinal and nominal variables. The paper then reviews and explores the numerical properties of these methods. An interesting finding is that the factor space can be quite precisely estimated directly from categorical data without quantification. This may require using a larger number of estimated factors to compensate for the information loss in categorical variables. Separate treatment of categorical and continuous variables may not be necessary if structural interpretation of the factors is not required, such as in forecasting exercises.
\end{abstract}

Keywords: factor models, principal components, ordinal data, alternating least squares.

JEL Classification: C5, C6, C25, C35

${ }^{*}$ Correspondence: 420 W. 118 St. New York, NY 10025. Email: serena.ng@columbia.edu

I thank Aman Ullah for teaching me econometrics and especially grateful for his guidance and support over the years. Comments from two anonymous referees are greatly appreciated. I also thank Nickolay Trendafilov for helpful comments and discussions. Financial support from the National Science Foundation (SES-0962431) is gratefully acknowledged. 


\section{Introduction}

The recent interest of economists in factor models is largely driven by the fact that common factors estimated from large panels of data often have predictive power for economic variables of interest. Theoretical and empirical work predominantly use principal components to estimate the common factors in continuous data. Little attention has been given to alternative estimators and to the treatment of categorical data even though many economic variables are of this nature. For example, households and firms are asked in surveys whether they expect economic conditions to improve or not. While such data could be useful for forecasting, they cannot be approximated by continuous distributions. This paper first reviews dimension reduction methods that can handle mixed measurement data, meaning that the data can be continuous or categorical. I then investigate the consequence from the perspective of the factor space of constructing principal components from categorical variables as if they were continuous.

Any study of socio-economic status necessarily involves analyzing dichotomous data or data with a small number of categories. Such data necessitate special treatment as they contain important but imperfect information about the underlying latent variables. Racine and $\mathrm{Li}$ (2004) and $\mathrm{Su}$ and Ullah (2009) consider using a small set of mixed data in non-parametric regressions. Here, I consider the situation when the set of mixed predictors is large enough that dimenional reduction becomes necessary. As pointed out in Kolenikov and Angeles (2009) and further investigated below, the method that is currently used by economists is far from satisfactory.

The psychometric approach to the dimension reduction problem is to either explicitly model the latent continuous variables or quantify (impute) the continuous variables from the categorical data. ${ }^{1}$ According to the SPSS software and as explained in Meulman and Heiser (2001), three types of categorical variables are relevant: - (1) nominal variables which represent unordered categories (such as zip codes and SIC codes); (2) ordinal variables which represent ordered categories (such as satisfaction ratings of excellent/good/average/poor and Likert scale), and (3) numerical (count) variables which represent ordered categories (such as age in years and income class in dollars) with distances between categories that can be meaningfully interpreted. Nominal and ordinal data are said to be non-metrical because the distance between two categories has no meaningful interpretation. The challenge for factor analysis of non-metrical data lies in the fact that normalization and monotonicity constraints need to be imposed to ensure consistency between the imputed variables and the observed discrete variables. Not surprisingly, going down this route necessarily takes us from linear to non-linear methods of dimension reduction.

I begin with a review of factor analysis of continuous data from the viewpoint of solving a

\footnotetext{
${ }^{1}$ An 1983 issue of Journal of Econometrics, (de Leeuw and Wansbeek editors) was devoted to these methods.
} 
Procrustes problem. These methods are non-probabilistic and do not permit formal inference to be made. But they form the basis of many dimension reduction problems which are interesting in their own right. The issues that arise in factor analysis of categorical data are then discussed. Special focus is given to methods that quantify the discrete data. Simulations are used to evaluate the precision of the factors estimated from continuous and mixed data. The so-called Filmer Pritchett procedure is also evaluated. I assess the factors estimates from the perspective of diffusion index forecasting which requires extracting common information in a large number of categorical variables. Precise estimation of the factor space rather than structural interpretation of the factor estimates takes center-stage. ${ }^{2}$ An interesting finding is that the principal components of the raw discrete data can estimate the factor space reasonably precisely, though this may require over-estimating the number of factors to compensate for the information loss in categorical data. Data quantification may not be necessary.

\section{Factor Analysis of Continuous Data}

Factor analysis is a statistical framework used to analyze the behavior of observed variables using a small number of unobserved factors. Spearman (1904) appears to be the first to use factor analysis. He observed that children's test scores on a variety of subjects were positively correlated and conjectured that a general unobserved trait (mental ability) is responsible for the observed data. To analyze the contribution of the factors on the test scores and more generally on data that are continuous, the classical approach is to estimate the factor loadings by maximizing the Gaussian likelihood. Important contributions have subsequently been made by Anderson and Rubin (1956), Joreskog (1970), Lawley and Maxwell (1971), Browne (1984), among others. Factor models are now used not just by psychologists, but by researchers in marketing, biology, and other fields.

Let $X$ denote a $T \times N$ matrix of continuous data or data in ratio form. As a matter of notation, the $i, j$ entry of $X$ is denoted $X_{i j} ; X_{i,:}$ is the $i$-th row of $X$ and $X_{:, j}$ is the $j$-th column. For macroeconomic panels, $N$ is the number of variables and $T$ is the number of time periods over which the variables are observed. A superscript zero is used to denote true values. The goal of factor analysis is to explain $X$ using $r$ common factors $F^{0}=\left(F_{1}^{0}, \ldots, F_{r}^{0}\right)$ and $N$ idiosyncratic errors $e^{0}$. In matrix form, the factor representation of the data is

$$
X=F^{0} \Lambda^{0 \prime}+e^{0}
$$

where $\Lambda^{0}$ is a $N \times r$ matrix of factor loadings. The population covariance structure of $X$ under the

\footnotetext{
${ }^{2}$ The focus is rather different from the structural factor analysis considered in Cunha and Heckman (2008); Almund, Duckworth, Heckman, and Kautz (2011).
} 
assumption that the factors have unit variance and are mutually uncorrelated is

$$
\Sigma_{X}^{0}=\Lambda^{0} \Lambda^{0 \prime}+\Omega^{0}
$$

In classical factor analysis, $\Omega^{0}$ is assumed to be a diagonal matrix and the data $X$ are said to have a strict factor structure. Let $k$ be the assumed number of factors which can be different from $r$. Anderson and Rubin (1956) assume that the data are multivariate normal. Using the normalization that $\Sigma_{F^{0}}=I_{r}$, they suggest to estimate the factor loadings by maximizing the log likelihood:

$$
\log L_{0}(\Lambda, \Omega ; k)=\log |\Omega|+\operatorname{trace}\left(X-F \Lambda^{\prime}\right) \Omega^{-1}\left(X-F \Lambda^{\prime}\right)^{\prime} .
$$

Lawley and Maxwell (1971) consider the equivalent problem of maximizing

$$
\log L_{1}(\Lambda, \Omega ; k)=\log \left|\Sigma_{X}\right|+\operatorname{trace}\left(S_{X} \Sigma_{X}^{-1}\right)-\log \left|S_{X}\right|-N
$$

where $S_{X}$ is the sample covariance of $X$. An advantage of maximum likelihood estimation is that the sampling distribution of $\widehat{\Lambda}$ is known and inference can be made. When $T$ is large and $N$ is fixed, the factor estimates $\widehat{\Lambda}$ are $\sqrt{T}$ consistent and asymptotically normal. Unbiased estimates of $F$ can be obtained as shown in Lawley and Maxwell (1971, Ch.8) even though these are not usually the object of interest in psychology research.

When the normality assumption is not appropriate, one alternative is to consider covariance structure estimation (also known as structural equation modeling). Let $\theta=(\operatorname{vec}(\Lambda) \text {, vech }(\Omega))^{\prime}$ be the parameters of the factor model. The weighted least squares estimator is

$$
\widehat{\theta}_{W L S}=\underset{\theta}{\operatorname{argmin}}\left(\operatorname { v e c h } ( \Sigma _ { X } ( \theta ) - \operatorname { v e c h } ( S _ { X } ) ) ^ { \prime } W ^ { - 1 } \left(\operatorname{vech}\left(\Sigma_{X}(\theta)-\operatorname{vech}\left(S_{X}\right)\right) .\right.\right.
$$

Under regularity conditions and assuming that $N$ is fixed, the WLS estimator is also $\sqrt{T}$ consistent and asymptotically normal, as shown in Browne (1984) and others. As $\widehat{\theta}_{W L S}$ is simply a method of moments estimator, it is less efficient than MLE but is robust to departures from normality. Assuming that the true number of factors is known, it has been documented that the asymptotic approximation of the WLS estimator is not accurate when the data exhibit excess kurtosis; the chi-square statistic for goodness of fit is oversized; the factor loadings are underestimated, and the standard error of the estimates tend to be downward biased.

Another alternative to MLE is iterative least squares estimation, the best known in this category being MINRES. Given a $N \times N$ sample correlation matrix $R_{X}$, the objective is to find a $N \times N$ matrix $\Omega$ and a $N \times k$ matrix $\Lambda$ such that $\Lambda \Lambda^{\prime}$ is as close to $R_{X}$ as possible. Formally,

$$
L_{M I N R E S}(\Lambda ; k)=\left\|R_{X}-\Lambda \Lambda^{\prime}-\Omega\right\|^{2} .
$$


Concentrating out $\Omega$ and using the fact that the diagonal entries of $R_{X}$ all equal one, the concentrated loss function is

$$
L_{M I N R E S}^{c}(\Lambda ; k)=\sum_{i \neq j}\left(R_{X, i j}-\Lambda_{i,:} \Lambda_{j,:}^{\prime}\right)^{2}
$$

where $\Lambda_{i, \text { : }}$ is the $i$-th row of $\Lambda$, and $R_{X, i j}$ is the $(i, j)$ entry of $R_{X}$. Harman and Jones (1966) suggest to start with an arbitrary $\Lambda$ and iterate on each row of $\Lambda$ holding other rows fixed. To update the rows, the objective function is separated into a part that depends on $\Lambda_{i, \text { : }}$ and a part $c_{i}$ that does not. Let $R_{X}^{-i}$ be the $i$-th column of $R_{X}$ with the $i$-element excluded, and let $\Lambda^{-i}$ be the $(N-1) \times k$ matrix of $\Lambda$ when the $i$-th row is deleted. Define

$$
\begin{aligned}
L_{M R S, i}^{c}\left(\Lambda_{i,:} ; k\right)= & \left(R_{X, 1 i}-\Lambda_{1,:} \Lambda_{i,:}^{\prime}\right)^{2}+\left(R_{X, i-1, i}-\Lambda_{i-1,:} \Lambda_{i,:}^{\prime}\right)^{2}+ \\
& \left(R_{X, i+1, i}-\Lambda_{i+1,:} \Lambda_{i,:}^{\prime}\right)^{2}+\ldots+\left(R_{X, k i}-\Lambda_{k,:} \Lambda_{i,:}^{\prime}\right)^{2}+c_{i} \\
= & \left\|R_{X}^{-i}-\Lambda^{-i} \Lambda_{i,:}^{\prime}\right\|^{2}+c_{i}
\end{aligned}
$$

The solution to this minimization problem is standard; it is the least squares estimate

$$
\widehat{\Lambda}_{i,:}^{\prime}=\left(\Lambda^{-i^{\prime}} \Lambda^{-i}\right)^{-1} \Lambda^{-i^{\prime}} R_{X}^{-i}
$$

Since all units other than $i$ are held fixed, the estimator is based on the principle of alternating least squares. Iterative updating of the $i$-th row of $\Lambda$ has been shown to decrease the loss function. However, a researcher would not be able to say whether a poor fit is due to the idiosyncratic errors or omitted factors. One shortcoming of MINRES is that $\widehat{\Lambda}_{i,:} \widehat{\Lambda}_{i,:}^{\prime}$ can exceed one, which would then imply a negative idiosyncratic error variance. This so-called Heywood case can be circumvented. An example is the LISREL implementation of MINRES, detailed in Joreskog (2003).

Strict (or exact) factor models are to be contrasted with models more widely used in macroeconomics and finance. These models relax the assumption of strict factor models to allow some cross-section and serial correlation in $e_{i t}$. Chamberlain and Rothschild (1983) referred to these as approximate factor models. Estimation is based on the method of asymptotic principal components (PCA) first proposed in Connor and Korajzcyk (1986). The estimator minimizes

$$
L_{P C A}(\Lambda, F ; k)=\frac{1}{N T} \sum_{i=1}^{N} \sum_{t=1}^{T}\left(x_{i t}-\Lambda_{i,:} F_{t}\right)^{2}=\frac{1}{N T} \sum_{i=1}^{N}\left(X_{:, i}-F \Lambda_{i}^{\prime}\right)^{\prime}\left(X_{:, i}-F \Lambda_{i}^{\prime}\right) .
$$

Because $\Lambda_{i,:}^{\prime}=\left(F^{\prime} F\right)^{-1} F^{\prime} X_{: i}$ for any given $F$, the concentrated objective function is:

$$
\begin{aligned}
L_{P C A}^{c}(F ; \Lambda ; k) & =\frac{1}{N T} \sum_{i=1}^{N}\left(X_{:, i}-P_{F} X_{:, i}\right)^{\prime}\left(X_{:, i}-P_{F} X_{:, i}\right) \\
& =\frac{1}{N T} \sum_{i=1}^{N} X_{:, i}^{\prime} X_{:, i}-\frac{1}{N} \sum_{i=1}^{N} X_{:, i}^{\prime} P_{F} X_{:, i}
\end{aligned}
$$


Since $F$ and $\Lambda$ are not separately identified, the normalization $F^{\prime} F / T=I_{k}$ or $\Lambda^{\prime} \Lambda / N=I_{k}$ is necessary. When $N>T$, minimizing $L_{P C A}^{c}(F ; \Lambda)$ subject to the constraint that $F^{\prime} F / T=I_{k}$ is the same as maximizing

$$
\operatorname{trace}\left(\frac{1}{N} \sum_{i=1}^{N} X_{:, i}^{\prime} F F^{\prime} X_{:, i}\right)=\operatorname{trace}\left(F^{\prime} \frac{1}{N} \sum_{i=1}^{N} X_{:, i} X_{:, i}^{\prime} F\right)=\frac{1}{N} \operatorname{trace}\left(F^{\prime} X X^{\prime} F\right) .
$$

The solution for $F$, denoted $\widehat{F}$, is $\sqrt{T}$ times the eigenvectors corresponding to the $k$ largest eigenvalues of the $T \times T$ matrix $X X^{\prime}$. The solution for $\Lambda$, denoted $\widehat{\Lambda}$, is $X^{\prime} \widehat{F} / T$. When $T>N$ and under the normalization that $\Lambda^{\prime} \Lambda / N=I_{k}, \widehat{\Lambda}$ is $\sqrt{N}$ times the eigenvectors corresponding to the $k$ largest eigenvalues of the $N \times N$ matrix $X^{\prime} X$ with $\widehat{F}=X \widehat{\Lambda} / N$. Note that unlike MLE and WLS which fix either $T$ or $N$, the sample sizes in both dimensions are allowed to be large so that inferential theory can be developed for both $\widehat{F}$ and $\widehat{\Lambda}$. Bai (2003) shows that if $\sqrt{N} / T \rightarrow 0$, then for each $t, \sqrt{N}\left(\widehat{F}_{t,:}^{\prime}-H F_{t,:}^{0 \prime}\right) \stackrel{d}{\longrightarrow} N\left(0, \operatorname{Avar}\left(\widehat{F}_{t}\right)\right)$, and if $\sqrt{T} / N \rightarrow 0$, then for each $i, \sqrt{T}\left(\widehat{\Lambda}_{i,:}^{\prime}-H^{-1} \Lambda_{i,:}^{0 \prime}\right) \stackrel{d}{\longrightarrow} N\left(0, \operatorname{Avar}\left(\widehat{\Lambda}_{i}\right)\right)$. As is well known, principal components describe but does not impose any structure on the data, while a factor model distinguishes between the common and idiosyncratic components. In a data rich environment, the distinction between factor models and principal components analysis is often left vague because the principal components consistently estimate the space spanned by the common factors.

A crucial assumption in the asymptotic theory for large dimensional factor models is that the largest eigenvalue of $\Omega^{0}$ is bounded. A sufficient condition is that $e_{i t}^{0}$ is weakly correlated across $i$ and $t$ because under stationarity, the largest eigenvalue of $\Omega^{0}$ is bounded by $\max _{j} \sum_{i=1}^{N}\left|E e_{i t}^{0} e_{j t}^{0}\right|$. Simulations show that $\widehat{F}_{t}$ precisely estimates the space spanned by $F_{t}^{0}$ when $\Omega^{0}$ is diagonal. This is a special case in which the eigenvalue bound is trivially satisfied. However, the construction of principal components as factor estimates do not take this constraint into account. Boivin and $\mathrm{Ng}$ (2006) report that estimated idiosyncratic errors associated with macroeconomic data tend to be quite pervasively cross-correlated. Onatski (2010) finds that asymptotic theory is a poor guide to the finite sample properties of the factor estimates when the errors are strongly cross-sectionally correlated. However, few alternatives to the PCA have been considered. Doz, Giannone, and Reichlin (2007) suggest a quasi-maximum likelihood approach that assumes $\Omega$ is diagonal even if the errors are serially and cross-sectionally correlated. A Kalman smoother is used to build up the likelihood which is then maximized using the EM algorithm. They find that omitting the correlation in the errors has negligible effects on the estimated common factors when $N$ is large. But their simulations only consider mildly correlated errors. 


\section{Two Alternating Least Squares Estimators (ALS)}

As alternatives to the method of principal components, I consider two estimators (ALS1 and ALS2) that address the Haywood problem of negative idiosyncratic error variances. ALS2 additionally allows us to assess if the common and idiosyncratic components are poorly estimated. As distinct from all estimators considered in the previous section, the idiosyncratic errors are explicitly recognized in the objective function, putting them on equal footing with the common factors. Furthermore, the two estimators solve for the factors without writing down the probability structure of the model and as such, the statistical properties of the estimates are not known. It may perhaps be inappropriate to call these estimators. My interest in these methods arises because they can be extended to study latent variable models for mixed (discrete and continuous) data.

Whereas the standard approach to deriving an estimator is to take derivatives of the objective function, a derivative free alternative is to exploit information in the objective function evaluated at the upper or lower bound. For example, consider finding the minimum of $f(x)=x^{2}-6 x+11$. By completing the squares and writing $f(x)=(x-3)^{2}+2$, it is clear that the lower bound for $f(x)$ is 2 and is achieved at $x=3$. Knowing the lower bound helps to determine the minimizer. ${ }^{3}$ ten Berge (1993) and Kiers (2002) provide a formal treatment of using bounds to solve matrix optimizaiton problems.

Lemma 1 Let $Y$ be of order $p \times q$ with singular value decomposition $Y=P D Q^{\prime}$. Let $d_{i}$ be the $i$-th diagonal value of $D$. Then (i) trace $\left(B^{\prime} Y\right) \leq \sum_{i=1}^{q} d_{i}$; and (ii) The maximum of trace $\left(B^{\prime} Y\right)$ subject to the constraint $B^{\prime} B=I$ is achieved by setting $B=P Q^{\prime}$.

Kristof's upper bound for trace functions states that if $G$ is a full rank orthonormal matrix and $D$ is diagonal, then the upper bound of trace $(G D)$ is $\sum_{i=1}^{n} d_{i}$. ten Berge (1993) generalizes the argument to sub-orthonormal matrices of rank $r \leq n$. Now trace $\left(B^{\prime} Y\right)=\operatorname{trace}\left(B^{\prime} P D Q^{\prime}\right)=$ trace $\left(Q^{\prime} B^{\prime} P D\right) .{ }^{4}$ Part (i) follows by letting $G=Q^{\prime} B^{\prime} P$. Part (ii) says that the upper bound under the orthogonality constraint is attained by setting $G=I$ or equivalently, $B=P Q^{\prime}$. This second result is actually the solution to the orthogonal 'Procrustes problem' underlying many dimension reduction problems and is worthy of a closer look. ${ }^{5}$

Let $A$ be a $n \times m$ matrix (of rank $r \leq m \leq n$ ) and let $C$ be a $n \times k$ matrix with a specified structure. The orthogonal Procrustes problem looks for an orthogonal matrix $B$ of dimension

\footnotetext{
${ }^{3}$ It is important that the lower bound is attainable and does not depend on $x$. If the lower bound was 1 instead of 2 , no meaning could be attached to $x=3$ because the lower bound of 1 is not attainable.

${ }^{4} \mathrm{~A}$ matrix is sub-orthonormal if it can be made orthonormal by appending rows or columns.

${ }^{5}$ The orthogonal Procrustes problem was solved in Schonmenn (1966). See Gower and Dijksterhuis (2004) for a review for subsequent work.
} 
$m \times k$ that closely maps $A$ to $C$. Formally, one chooses $B$ to minimize $\|C-A B\|_{F}^{2}$ subject to the constraint $B^{\prime} B=I_{m} \cdot{ }^{6}$ By definition,

$$
\|C-A B\|_{F}^{2}=\operatorname{trace}\left(C^{\prime} C+B^{\prime} A^{\prime} A B-2 B^{\prime} A^{\prime} C\right) .
$$

When $A$ has the same number of columns as $C, B$ must be a $k \times k$ orthonormal matrix satisfying $B^{\prime} B=B B^{\prime}=I_{k}$. The first two terms do not depend on $B$ since trace $\left(B^{\prime} A^{\prime} A B\right)=$ trace $\left(B B^{\prime} A^{\prime} A\right)=$ trace $\left(A^{\prime} A\right)$. The minimization problem is thus the same as maximizing trace $\left(B^{\prime} A^{\prime} C\right)$ subject to the constraint that $B^{\prime} B=I$. Letting $P D Q^{\prime}$ be the singular value decomposition of $A^{\prime} C$ gives trace $\left(B^{\prime} A^{\prime} C\right)=\operatorname{trace}\left(B^{\prime} P D Q^{\prime}\right)=\operatorname{trace}\left(Q^{\prime} B^{\prime} P D\right)$. Since $Q^{\prime} B^{\prime} P$ is orthogonal and $D$ is a diagonal positive matrix, the trace is maximized if $Q^{\prime} B^{\prime} P$ is an identity matrix. The solution is to set $B=P Q^{\prime}$. When $m>k$ and $B$ is a long matrix with more rows than columns, the solution has no closed form and must be solved by iteration.

To see how Lemma 1 can be used to construct principal components, I follow the formulation in ten Berge (1993). Given a $T \times N$ matrix of standardized data $X$, the goal of principal components analysis is to find $k$ linear combinations given by the $N \times k$ matrix $B$ such that $F=X B$ optimally summarizes information in $X$. Let $A^{\prime}$ be the weights for estimating $F$ from $X$. The loss function

$$
L_{P C A}^{c}(B, A)=\left\|X-F A^{\prime}\right\|^{2}
$$

is to be minimized subject to $F^{\prime} F / T=B^{\prime}\left(X^{\prime} X / T\right) B=B^{\prime} R_{X} B=I_{k}$. Concentrating out $A^{\prime}=$ $\left(F^{\prime} F\right)^{-1} F X$ and defining $Y=R_{X}^{1 / 2} B$ gives

$$
\begin{aligned}
L_{P C A}^{c}(B ; A) & =\left\|X-X B\left(F^{\prime} F\right)^{-1} F^{\prime} X\right\|^{2}=\left\|X-X B B^{\prime} R_{X}\right\|^{2} \\
& =\operatorname{trace}\left(X^{\prime} X\right)-2 T \operatorname{trace}\left(B^{\prime} R_{X}^{2} B\right)+T \cdot \operatorname{trace}\left(B^{\prime} R_{X}^{2} B\right) \\
& =T \cdot k-T \cdot \operatorname{trace}\left(B^{\prime} R_{X}^{2} B\right) \\
& =T \cdot k-T \cdot \operatorname{trace}\left(Y^{\prime} R_{X} Y\right) .
\end{aligned}
$$

The constrained minimization problem now becomes maximizing trace $\left(B^{\prime} R_{X} B\right)$ subject to $B^{\prime} R_{X} B$, or equivalently, maximizing trace $\left(Y^{\prime} R_{X} Y\right)$ subject to $Y^{\prime} Y=I_{k}$. From the eigen-decomposition $R_{X}=P D P^{\prime}$,

$$
\text { trace }\left(Y^{\prime} R_{X} Y\right)=\operatorname{trace}\left(Y^{\prime} P D P^{\prime} Y\right)=\operatorname{trace}\left(P^{\prime} Y Y^{\prime} P D\right)=\operatorname{trace}(G D)
$$

with $G=P^{\prime} Y Y^{\prime} P$. The upper bound of trace $(G D)=\sum_{i=1}^{k} d_{i}$ is achieved if $G$ is sub-orthonormal, ie. $G=\left(\begin{array}{cc}I_{k} & 0 \\ 0 & 0\end{array}\right)$. We thus let $Y=P_{:, 1: r}$ and $B=P_{:, 1: k} D_{1: k, 1: k}$ (times any $k \times k$ orthonormal matrix). Canonical correlation analysis and many constrained least squares problems with no closed form solutions can also be solved using these monotonically convergent algorithms.

\footnotetext{
${ }^{6}$ For a $p \times q$ matrix $A,\|A\|_{F}=\operatorname{trace}\left(A A^{\prime}\right)^{1 / 2}=\sqrt{\sum_{i=1}^{p} \sum_{j=1}^{q} A_{i j}^{2}}$.
} 


\subsection{ALS1}

I first consider the estimator proposed in De Leeuw (2004), Unkel and Trendafilov (2010) and Trendafilov and Unkel (2011). Let $e=u \Psi$ where $u^{\prime} u=I_{N}$. Let $k$ be the number of assumed (not necessarily equal $r$, the true) number of factors. Suppose first that $T \geq N$ and consider maximizing

$$
\begin{aligned}
& L_{A L S 1}(F, \Lambda, u, \Psi ; k)=\left\|X-F \Lambda^{\prime}-u \Psi\right\|_{F}^{2} \\
& \text { subject to } \quad(i) F^{\prime} F=I_{k}, \quad(i i) u^{\prime} u=I_{N}, \quad(i i i) u^{\prime} F=0_{N \times k}, \quad \text { (iv) } \Psi \text { is diagonal. }
\end{aligned}
$$

Notice that ALS minimizes the difference between the $X$ and its fitted value $\widehat{X}=\widehat{F} \widehat{\Lambda}^{\prime}+\widehat{e}$. While the idiosyncratic and the common components are explicitly chosen, distributional assumptions on $e$ are not made. Furthermore, the objective function takes into account the off-diagonal entries of the fitted correlations. In contrast, the PCA loss function only considers the diagonal entries of $(X-F \Lambda)^{\prime}\left(X-F \Lambda^{\prime}\right)$. Define

$$
\underset{T \times(k+N)}{\stackrel{B}{\leftrightarrows}}=\left(\begin{array}{ll}
F & U
\end{array}\right), \quad \underset{N \times(k+N)}{A}=\left(\begin{array}{ll}
\Lambda & \Psi
\end{array}\right) .
$$

The ALS estimates are obtained by minimizing

$$
L_{A L S 1}(B, A ; k)=\left\|X-B A^{\prime}\right\|_{F}^{2} \quad \text { subject to } B^{\prime} B=I_{N+k} .
$$

But for given $A$, this is the same as maximizing trace $\left(B^{\prime} X A\right)$ over $B$ satisfying $B^{\prime} B=I_{N+k}$. The problem is now in the setup of Lemma 1 . When $N \leq T$, the estimates $(\widetilde{F}, \widetilde{\Lambda}, \widetilde{U})$ can be obtained using the following three step procedure:

1 Let $\widetilde{B}=P Q^{\prime}$ where $\operatorname{SvD}(X A)=P D Q^{\prime}$.

2 From $\widetilde{B}=\left(\widetilde{B}_{:, 1: k} \mid \widetilde{B}_{:, k+1: k+N}\right)$, let $\widetilde{F}=\widetilde{B}_{:, 1: k}$ and $\widetilde{U}=\widetilde{B}_{:, k+1: N}$. Update $\widetilde{\Lambda}$ as $X^{\prime} \widetilde{F}$.

3 Let $\widetilde{\Psi}=\operatorname{diag}\left(\widetilde{U}^{\prime} X\right)$.

Steps (1) and (2) are based on part (ii) of Lemma 1. Step (3) ensures that $\Psi$ is diagonal and is motivated by the fact that

$$
U^{\prime} X=U^{\prime} F \Lambda^{\prime}+U^{\prime} U \Psi
$$

Steps (1)-(3) are repeated until the objective function does not change. As $F \Lambda^{\prime}$ is observationally equivalent to $F C^{-1} C \Lambda^{\prime}$ for any orthogonal matrix $C$, Step (2) can be modified to make the top $k \times k$ sub-matrix of $\Lambda$ lower triangular. This identification restriction does not affect the fact that $\operatorname{rank}(\Lambda)=\min (\operatorname{rank}(X), \operatorname{rank}(F))=k$.

When $N>T$, the rank of $U^{\prime} U$ is at most $T$ and the constraint $U^{\prime} U=I_{N}$ cannot be satisfied. However, recall that $\Sigma_{X}=\Lambda \Lambda^{\prime}+\Psi U^{\prime} U \Psi$. Trendafilov and Unkel (2011) observe that the population 
covariance structure of the factor model can still be preserved if the constraint $\Psi U^{\prime} U=\Psi$ holds, and in that case, $\Omega=\Psi^{2}$ is positive semi-definite by construction. Furthermore, the constraints $F^{\prime} F=I_{k}$ and $U^{\prime} F=0_{N \times k}$ are equivalent to $F F^{\prime}+U U^{\prime}=I_{T}$ and $\operatorname{rank}(F)=k$. Minimizing

$$
L_{A L S 1}(B, A ; k)=\left\|X-B A^{\prime}\right\|_{F}^{2} \quad \text { subject to } \quad B B^{\prime}=I_{T}
$$

is the same as maximizing trace $\left(B A^{\prime} X^{\prime}\right)$ which is again a Procrustes problem. The three step solution given above remains valid when $N>T$, but the singular value decomposition in step (a) needs to be applied to $A^{\prime} X^{\prime}$.

Trendafilov and Unkel (2011) show that the objective function will decreases at each step and the algorithm will converge from any starting value. However, convergence of the objective function does not ensure convergence of the parameters. Furthermore $B$ is not unique because it is given by the singular value decomposition of a rank deficient matrix. In particular, $\operatorname{rank}(X A) \leq$ $\min (\operatorname{rank}(X), \operatorname{rank}(A))<\operatorname{rank}(X)+k$.

\section{$3.2 \quad$ ALS2}

To motivate the second estimator, partition $F^{0}$ as $F^{0}=\left(\begin{array}{ll}F^{01} & F^{02}\end{array}\right)$ where $F^{01}$ has $k$ columns and $F^{02}$ has $r-k$ columns for some $k<r$. The data generating process can be written as

$$
X=F^{01} \Lambda^{1 \prime}+F^{02} \Lambda^{2 \prime}+e^{0}=F^{01 \prime} \Lambda^{1}+e^{*} .
$$

If $k<r$ factors are assumed, the omitted factors $F^{02}$ will be amalgamated with $e^{0}$ into $e^{*}$. Even though $\Omega^{0}$ is diagonal in the population, the off-diagonal entries of the covariance matrix for $e^{*}$ could be non-zero. Without explicitly imposing the restriction that $X$ and $e^{0}$ are orthogonal, an estimator may confound $e^{0}$ with $F_{2}^{0}$. Socan (2003) re-examined an (unpublished) idea by H. Kiers to minimize

$$
\begin{aligned}
L_{A L S 2}(F, \Lambda, e ; k)= & \left\|X-F \Lambda^{\prime}-e\right\|_{F}^{2} \\
\text { subject to } \quad & (i) e^{\prime} F=0 \quad(i i) e^{\prime} e \text { diagonal } \quad(i i i) e^{\prime} X \text { diagonal. }
\end{aligned}
$$

As with ALS1, estimation of $e$ is explicitly taken into account. The first two constraints are standard; the third constraint ensures that the idiosyncratic errors are truly uncorrelated across units. This is because given orthogonality between $e$ and $F$, any non-zero correlation between $e_{i t}$ and $x_{j t}$ when $i \neq j$ can only arise if $e_{i t}$ is a function of the omitted factors.

The estimates $(\bar{F}, \bar{\Lambda}, \bar{e})$ are iteratively updated until the objective function does not change.

1 Given $\bar{\Lambda}$, let $\bar{J}$ be in the orthogonal null space of $\bar{e}$ so that $\bar{F}=\bar{J} \bar{C}$ satisfies the constraints for $\bar{C}=J^{\prime} X \Lambda\left(\Lambda^{\prime} \Lambda\right)^{-1}=\min _{C}\left\|J C \Lambda^{\prime}-X\right\|^{2}$. 
2 For $i=1, \ldots N$, update $\bar{e}_{:, i}$ :

a Let $\left(X^{-i}, \bar{e}^{-i}\right)$ be $(X, \bar{e})$ with the $i$-th column set to zero.

b Let $H_{i}=\left(\begin{array}{lll}X^{-i} & F & \bar{e}^{-i}\end{array}\right)$ with $\operatorname{svd}\left(H_{i}\right)=P_{H i} D_{H i} Q_{H i}^{\prime}$,

c Let $\bar{e}_{:, i}=P_{H i}^{0} P_{H i}^{0 \prime} X_{:, i}$ where $P_{H i}^{0}$ is the last column of $P_{H i}$.

3 Update $\bar{\Lambda}=X^{\prime} \bar{F}\left(\bar{F}^{\prime} \bar{F}\right)^{-1}$.

Steps (1) and (3) are intuitive as linear combinations of the basis in the orthonormal null space of $e$ will be orthogonal to $e$. To find the optimal linear combinations, $C$ is chosen to minimize $\left\|X-F \Lambda^{\prime}\right\|^{2}=\left\|X-J C \Lambda^{\prime}\right\|^{2}$. This yields

$$
\bar{C}=\left(\bar{J}^{\prime} \bar{J}\right)^{-1} \bar{J}^{\prime} X \bar{\Lambda}\left(\bar{\Lambda}^{\prime} \bar{\Lambda}\right)^{-1}=\bar{J}^{\prime} X \bar{\Lambda}\left(\bar{\Lambda}^{\prime} \bar{\Lambda}\right)^{-1}
$$

Step (2) is more involved. Recall that for each $i$, I need to find a $\bar{e}_{:, i}$ that it is uncorrelated with $F$ and with $X_{:, j}$ and $\bar{e}_{:, j}$ for $j \neq i$. As rank $\left(H_{i}\right)$ is less than its column dimension, $P_{H i}^{0}$ is orthogonal to all but the $i$-th column of $X$ and $e$ as well as $F$. It remains to find the relation between $e_{:, i}$ and $P_{H i}^{0}$. Minimizing $\left\|X_{, i}-P_{H i}^{0} \beta_{i}\right\|$ yields $\bar{\beta}_{i}=P_{H i}^{0 \prime} X_{:, i}$. The update is thus $\bar{e}_{:, i}=P_{H i}^{0} \bar{\beta}_{i}=P_{H i}^{0} P_{H i}^{0 \prime} X_{:, i}$.

\section{Factor Analysis of Categorical Variables}

Suppose the continuous data $X$ are well represented by the factor model $X=F \Lambda^{\prime}+e$ but we observe $x$ which are discrete (nominal, ordinal, or numerical) transformations of $X$. Discrete data tend to be skewed and have excess kurtosis especially when the frequencies of observing a few categories are disproportionately high. Classical factor analysis is not appropriate because the normality assumption and Pearson correlation coefficients take as given that the data are continuous. As Kruskal and Shepard (1974) point out, a non-metrical factor analysis needs to recover data that can be represented with the smallest number of factors and yet be consistent with the observed categorical data. Several approaches have been considered.

\subsection{Probabilistic Approaches}

The Bayesian approach is to parametrically specify the factor representation for $X$ as well as the relation between $X$ and $x$. This permits joint estimation of the factors and the loadings, as in Conti, Heckman, Lopes, and Piatek (2012). Amongst the frequentist approaches, an 'item response' analysis specifies the conditional distribution of the responses as a function of the latent factors assuming that the responses are independent conditional on the factors. A frequentist method that does not specify the mechanism that generates $X$ is the 'underlying variable approach'. It 
simply assumes that each observed ordinal variable $x_{j}$ is generated by a normally distributed latent variable $X_{j}$ :

$$
x_{j}=a_{j} \quad \tau_{j} \leq X_{j} \leq \tau_{j-1}, \quad j=1, \ldots C_{j}-1
$$

where $\tau=\left(\tau_{1}, \ldots, \tau_{C_{j}-1}\right)$ is a vector of $C_{j}-1$ thresholds that define $C_{j}$ categories. Both the item response and the underlying variable approach requires calculations of tetrachoric or polychoric correlations. ${ }^{7}$ These are correlations between two continuous variables underlying the discrete indicators. Once they are computed, the likelihood or method of moments estimation can be used. Muthén (1984) proposes a three step estimator of the latent variable model as follows.

1. standardize $X$ to be mean zero, unit variance, and estimate the vector of thresholds $\tau$ from the univariate margins of the observed data.

2. estimate the polychoric correlations from the bivariate margins of the observed variables given the thresholds estimates. Denote the matrix of polychoric correlations by $\widehat{\Sigma}_{X}$;

3. Estimate $\theta$ (the factor loadings and variances) by maximizing the log likelihood (expressed in terms of the observed frequencies) or by minimizing the function

$$
\operatorname{vech}\left(\widehat{\Sigma}_{X}-\Sigma_{X}(\theta)\right)^{\prime} W^{-1} \operatorname{vech}\left(\widehat{\Sigma}_{X}-\Sigma_{X}(\theta)\right)
$$

with respect to $\theta$, where $W$ is a positive definite weighting matrix.

Compared to the method WLS described earlier for continuous data, parametric assumptions and an additional step of estimating the polychoric correlations are necessary. Joreskog and Moustki (2001) compare the latent variable and item response methods and find that full information methods are not very practical even though they are theoretically appealing. These estimators are designed for ordinal data; they can be computationally demanding even when $N$ is small. Fortunately, they are implemented in packaged software like LISREL, Joreskog and Sorbom (2006); Joreskog (1994).

The properties of these estimators applied to ordered data are documented in Babakus, Ferguson, and Joreskog (1987), Muthen and Kaplan (1985), Dolan (1994), Bollen (1989, Ch. 9), among others. The bias in the estimated loadings, while often less than $10 \%$ or so, depends on how far

\footnotetext{
${ }^{7}$ Suppose that two continuous variables $X_{1}$ and $X_{2}$ are jointly normal with correlation coefficient $\rho$. The probability that $\left(X_{1}>\tau_{1}, X_{2}>\tau_{2}\right)$ is given by

$$
p_{12}(\rho)=\frac{1}{2 \pi \sqrt{1-\rho^{2}}} \int_{\tau_{1}}^{\infty} \int_{\tau_{2}}^{\infty} \exp \left(-\frac{y_{1}^{2}+y_{2}^{2}-2 \rho y_{1} y_{2}}{2\left(1-\rho^{2}\right)}\right) d y_{1} d y_{2} .
$$

The tetrachoric correlation proposed by Pearson (1900), is the $\rho$ such that $p_{12}(\rho)$ equals the sample proportion $\widehat{p}_{12}$. Polychoric correlations are then generalizations of tetrachoric correlations from two dichotomous indicators to multiple ordered class.
} 
are the categorized data from normality. Furthermore, the covariance matrix of the estimated idiosyncratic errors often have non-zero off-diagonal entries even if the true idiosyncratic errors are mutually uncorrelated. The possibility that categorical data may lead to spurious factors has also been raised, McDonald (1985, Ch. 4).

\subsection{FACTALS and Optimal Scaling}

Methods have also been developed to estimate common factors in categorical data without specifying a probability model. Let $X$ be a $N \times J$ matrix and let $x$ denote the observed but discretized values of $X$. Let $\Lambda$ be a $N \times r$ matrix of factor loadings, $\Omega=D^{2}$ be a diagonal matrix of idiosyncratic error variances. If all $J$ columns of $X$ were continuous, the factor model implies the correlation structure $\Lambda \Lambda^{\prime}+\Omega(D)$. MINRES then minimizes

$$
L_{M I N R E S}(\Lambda, D ; r)=\left\|R_{X}-\Lambda \Lambda^{\prime}-\Omega(D)\right\|^{2} .
$$

As pointed out earlier, the minimization problem can be solved columnwise because $R_{X}$ is a correlation matrix. Now instead of $X$, a $N \times J$ matrix of data $x$ is observed, some columns are of $X$ continuous and some are discrete. The challenge for non-metrical factor analysis is that the discrete nature of the observables put constraints on the factor estimates. For example, to respect the fact that ordinal data are ordered, Kruskal and Shepard (1974) suggest to construct principal components subject to monotonicity constraints. Takane, Young, and de Leeuw (1979) argue that this does not fully exploit the factor structure. They suggest to replace $R_{X}$ by $R_{Z}$ where $Z$ is a $N \times J$ matrix of optimally scaled values of $x$.

Optimal scaling is an integral part of dimension reduction methods for non-metrical variables. Let $C_{j}$ be the number of categories in variable $j$ and let $G_{j}$ be a $N \times C_{j}$ indicator matrix that is one if variable $j$ is categorical with columns following the ordering of the categories. The adjacency matrix is $G=\left[G_{1}, G_{2}, \ldots G_{J}\right]$. The quantified scores for variable $j$ is $Z_{j}=G_{j} Y_{j}$ where $Y_{j}$ is estimated subject to constraints of the measured data. For example, if $x_{:, j}$ is ordinal, the restriction that $Y_{j}(1) \geq Y_{j}(2) \geq \ldots Y_{j}\left(C_{j}\right)$ is required. The exercise is to iteratively estimate $Y, \Lambda$ and $\Omega$ by minimizing

$$
L_{Q F A C}(\Lambda, D, Z ; k)=\left\|Z^{\prime} Z-\Lambda \Lambda^{\prime}-\Omega(D)\right\|^{2}
$$

subject to

$$
\text { i) } 1^{\prime} Z_{:, j}=0 \quad \text { (ii) } Z^{\prime} Z=I_{J} \quad \text { (iii) measurement level constraints }
$$

Note that each column of $Z$ is normalized to have unit sum of squares. The $Z^{\prime} Z$ matrix plays the same role as the sample correlation matrix. Note that this MINRES objective function is is defined for the quantified data $Z$ and not $X$, and $Z$ is being estimated. In a sense, the analysis proceeds as though $Z_{:, j}$ has factor representation $Z_{:, j}=F \Lambda_{j,:}^{\prime}+e_{:, j}$. 
While the problem seems conceptually simple, it is not trivial computationally because this is a quadratic program with a quadratic constraint (in view of the normalization for $Z^{\prime} Z$ ). Kiers, Takane, and Mooijaart (1993) propose a monotonically convergent FACTALS (factor analysis by alternating least squares) algorithm for estimating $k$ factors as follows. ${ }^{8}$

1 Let $\widehat{\Lambda}=U_{:, 1: k} S_{k}^{1 / 2}$ where $Z^{\prime} Z-\Omega$ has singular value decomposition $U S V$, with $U_{:, 1: k}$ being the first $k$ columns of $U$;

2 Let $\widehat{\Omega}=\operatorname{diag}\left(Z^{\prime} Z-\widehat{\Lambda} \widehat{\Lambda}^{\prime}\right)$ constrained to be non-negative;

3 Update $\widehat{R}_{Z}=\widehat{\Lambda} \widehat{\Lambda}^{\prime}+\widehat{\Omega}$. For $j=1, \ldots, J$ :

a if $x_{:, j}$ is continuous, $Z_{:, j}=x_{:, j}$. let $Z_{:,-j}$ be $Z$ with the $j$-th column removed, and $\widehat{R}_{Z,:,-j}$ be the $j$-th column of $\widehat{R}_{Z}$ with the $j$-th element removed.

b if $x_{j,:}$ is nominal, minimize $\left\|Z_{:,-j}^{\prime} G_{j} y_{j}-\widehat{R}_{Z,:,-j}\right\|^{2}$ subject to the constraint that $G_{j} y_{j}$ is centered and $y_{j}^{\prime} G_{j}^{\prime} G_{j} y_{j}=1$. Given the solution $y_{j}^{0}$, update $Z_{:, j}=G_{j} y_{j}^{0}$.

c if $x_{:, j}$ is ordinal, let $z=G_{j} y_{j}^{0}+a^{-1} Z_{:,-j} \widehat{R}_{Z,:-, j}-a^{-1} Z_{:,-j} Z_{:,-j}^{\prime} G_{j} y_{j}^{0}$ and minimize $\left\|z-G_{j} y_{j}\right\|^{2}$ subject to the constraints that (i) $G_{j} y_{j}$ is centered, (ii) $y_{j}^{\prime} G_{j}^{\prime} G_{j} y_{j}=1$, and (iii) the elements of $y_{j}$ are weakly ordered. Given the solution $y_{j}^{0}$, update $Z_{:, j}=G_{j} y_{j}^{0}$.

4 Check if $\left\|Z^{\prime} Z-\widehat{R}_{Z}\right\|^{2}$ converges. If not, return to step (1).

The thrust of FACTALS is to iteratively choose the scale values $y_{j}$ to yield the quantified data $Z_{j}$ and to update $\Lambda$ and $\Omega$. The first two steps perform columnwise update along the lines of MINRES. Step 3 imposes measurement level restrictions. Depending on the data type, it involves either solving an oblique Procrustes problem or performing a monotone regression. In a sense, FACTALS is a data augmentation method that treats $X$ as missing values and imputes them as $Z$. These steps are further explained in the Appendix.

\subsection{Principal Component Analysis}

While economists rarely consider principal component analysis of qualitative data, the literature on this problem is in fact large. As surveyed in Michailidis and de Leeuw (1998), seemingly related research appears under a variety of names:- homogeneity analysis, multiple correspondence analysis, PRINCALS systems, PRINCIPALS, discriminant analysis, to name a few. ${ }^{9}$ Related approaches

\footnotetext{
${ }^{8}$ The initial procedure proposed by Takane, Young, and de Leeuw (1979) and refined by Nevels (1989) both have shortcomings. FACTALS fixes those bugs. Special thanks to H. Kiers for sharing the MATLAB code.

${ }^{9}$ The method has been discovered and rediscovered under different names, including as quantification, multiple correspondence analysis, dual or optimal scaling and homogeneity analysis. See Tenenhaus and Young (1985) for a synthesis of these procedures. However, none of these methods are familiar to economists.
} 
also include the principal factor analysis of Keller and Wansbeek (1983) and redudancy analysis (canonical correlation) of Israels (1984). As with continuous data, principal component analysis differs from factor analysis by going a step further to impose a structure.

Recall that given a $T \times N$ matrix of standardized continuous variables standardized to $X$, PCA computes $\widehat{\Lambda}=T^{-1} X^{\prime} F$ where $F$ is a $T \times r$ matrix of common components. PCA can be generalized to mixed data as follows. If variable $j$ in the mixed data set is quantitative, let

$$
S_{j}=\frac{1}{T} X_{:, j} X_{:, j}^{\prime}
$$

be the quantification matrix where $X_{:, j}$ contains the standardized values of the $T$ observations on variable $j$. If variable $j$ is qualitative, the quantification matrix is defined as

$$
S_{j}=M G_{j} D_{j}^{-1} G_{j} M
$$

where $M=I-11^{\prime} / T$ is the centering matrix, $D_{j}$ is a diagonal matrix of frequencies of the categories in variable $j, G_{j}$ is the $T \times C_{j}$ indicator matrix for variable $j$. A principal components of mixed data then minimizes

$$
\sum_{j=1}^{N} \operatorname{trace} F^{\prime} S_{j} F
$$

over $F$ subject to $F^{\prime} F / T=I_{r}$ where the $T \times r$ matrix $F$ contains the standardized values of the components. The solution is given by the first $r$ eigenvectors of $\sum_{j} S_{j}$. If all variables are quantitative, the loadings are the eigenvectors of $X^{\prime} X / T$ which is the PCA solution. If all variables are qualitative, the solution is the eigenvectors of $\sum_{j} M G_{j} D_{j}^{-1} M$. Sophisticated methods go one step further to impose level constraints (as in FACTALS) and may also allow for multiple quantification. See, for example, the PRINCIPALS routine in SAS and R, PRINCALS in R (HOMALS), and SPSS (CATPCA). I explore these methods in on-going empirical work but do consider them in simulations as these procedures are well studied in statistics and the psychometrics literature.

An approach that has gained popularity in analysis of socio-economic data is the so-called Filmer-Pritchett method used in Filmer and Pritchett (1998). Essentially, the method constructs principal components from the adjacency matrix, G. Kolenikov and Angeles (2009) assess the model's predicted rankings and find the method to be inefficient because it loses the ordinal information in the data. Furthermore, spurious negative correlation in the $G$ matrix could lead to underestimation of the common variations in the data. However, they also find that constructing principal components from polychoric correlations of socio-economic data did not yield substantial improvements over the Filmer-Pritchett method.

I explore two alternatives that seem sensible when structural interpretation of the factors is not needed as in economic forecasting. The first is to ignore the fact that some variables are actually 
discrete. To grasp the motivation, note first that the population covariance of the categorical variables $x$ (ie $\Sigma_{x}$ ) will be different from the population covariance of the continuous variables $X$ (ie. $\Sigma_{X}$ ). An estimate of the $(i, j)$-th entry of $\Sigma_{x}$ obtained by regressing $x_{:, i}$ on $x_{:, j}$. will be biased for the corresponding entry in $\Sigma_{X}$ as $x$ is $X$ measured with errors. Lancaster (1957) shows that if a bivariate distribution is obtained by separate transformations of $Y$ and $Z$ that are bivariate normal, then the correlation of the transformed distribution cannot exceed the correlation coefficient of $\rho_{Y Z}$ in the bivariate normal distribution. ${ }^{10}$ In fact, discretization is a form of data transformation that is known to reduce the linear relation between $Y$ and $Z$, though the problem is alleviated as the number of categories increases. As mentioned earlier, many simulation studies have found that the $r$ factor loadings estimated by MLE and WLS do not behave well when the data exhibit strongly non-Gaussian features. These findings fit the description of the problem induced by data transformations.

Suppose I am only interested in dimension reduction of the categorical variables for forecasting. The exercise does not require structural interpretation of the factor estimates as what matters is that the space spanned by the factors can be precisely estimated from the data. As a example, suppose that $X=F^{0} \Lambda^{0}+e^{0}, F_{t}^{0}$ is a scalar, $\Lambda^{0}$ is a unit vector, and $e_{i t}^{0} \sim N(0,1)$ for all $i$ and $t$. If $X$ were observed, the principal component estimate is $\widehat{F}_{t}=\frac{1}{N} \sum_{i=1}^{N} X_{i t}-\bar{X}_{i,:}$. We only observe $x_{i t}$ which is one when $X_{i t}-\bar{X}_{:, t}$ is positive and minus one otherwise. The principal component estimate is $\widetilde{F}_{t}=\frac{1}{N} \sum_{i=1}^{N} x_{i t}-\bar{x}_{i,:}$. Given the assumed homogeneity of $\Lambda^{0}, x_{i t}-\bar{x}_{:, t}$ merely reflects crosssectional variations in the idiosyncratic errors. In this example, $\widetilde{F}_{t}$ has no information about $F_{t}^{0}$ whatever is $N$. But with more categories, heterogeneous factor loadings, and different positioning of the thresholds, $x_{i t}-\bar{x}_{i,:}$ should preserve some information about $F_{t}^{0}$. The hope is that that principal components will be able to extract this information.

The second method I consider can be motivated as follows. If principal components precisely estimates the space spanned by $X$, and $Z$ are good quantifications of the discrete data $x$, then PCA applied to $Z$ should estimate the space spanned by the common factors in $X$. I therefore obtain the quantified data $\widehat{Z}$ by FACTALS and then apply PCA to the covariance of $\widehat{Z}$ (ie. $R_{\widehat{Z}}$ ) to obtain estimate $\widehat{F}$ and $\widehat{\Lambda}$. The approach is experimental as I am unaware of estimating the factors from categorical data this way. However, it is an intuitive generalization of the method of asymptotic principal components. The $\widehat{\Lambda}$ estimated by PCA will generally be different from those that directly emerge from FACTALS because PCA does not impose diagonality of $\Omega$.

\footnotetext{
${ }^{10}$ Olsson, Drasgow, and Dorans (1982) show that $\rho_{Y z}$ is downward biased for $\rho_{Y Z}$ if $Y$ and $Z$ are jointly normal. The greatest attenuation occurs when there are few categories and the data are opposite skewed. In the special case when consecutive integers are assigned to categories of $Y$, it can be shown that $\rho_{Y z}=\rho_{Y Z} \cdot q$, where $\quad q=\frac{1}{\sigma_{\theta}} \sum_{j=1}^{J-1} \phi\left(\alpha_{j}\right)$ and $\phi(\cdot)$ is the standard normal density and $q$ is the categorization attenuation factor.
} 


\section{Monte Carlo Simulations}

This section has two sections. The first subsection focuses on continuous data and assesses the precision of the factor estimates produced by the method of asymptotic principal components, ALS1, and ALS2. I also evaluate criterion for determining the number of factors. Subsection two turns to categorical and mixed data. All computations are based on MATLAB Release 2011a.

\subsection{Continuous Data}

Data with macroeconomic characteristics are generated from an approximate factor model. Specifically, two serially correlated factors are assumed with $\rho_{F k} \sim U(0, .8)$ for $k=1, \ldots r$ and

$$
\begin{array}{rlrl}
X_{i t} & =\Lambda_{i,:}^{0} F_{t}^{0}+e_{i t} & \Lambda_{i, j}^{0} \sim N\left(0, \theta^{2}\right), & \theta^{0}=\{1, .5\} \\
F_{k, t}^{0} & =\rho_{F, k}^{0} F_{k, t-1}^{0}+u_{k t}, & u_{k t} \sim N(0,1) \\
e_{i t} & =\rho_{e}^{0} e_{i t-1}+\varepsilon_{i t}, & \varepsilon_{t,:}^{\prime} \sim N(0, I) C^{0}
\end{array}
$$

with $\rho_{e} \sim U(0, .8)$. The degree of cross-section correlation in errors is deteremined by

$$
C^{0}=\operatorname{toeplitz}\left(\left[1, u_{1 \times N_{c}}, 0_{1 \times N-N_{c}-1}\right]\right)
$$

where $N_{c}=\{0, .1 N\}$. As $\Omega^{0}=C^{0} C^{0 \prime}$, the number of correlated series is much larger than $.1 N$. The common component is strong when $\theta^{0}=1$ and weak if $\theta^{0}=.5$, all else equal. Since $\Lambda^{0}$ is drawn randomly, $F_{1}^{0}$ is not necessarily more important than $F_{2}^{0}$. The relative importance of the common component in the population is given by

$$
\text { SIGNAL }=\sum_{s=1}^{S} \operatorname{SIGNAL}^{s}, \quad \operatorname{SIGNAL}^{s}=1-\frac{\sum_{i=1}^{N} \operatorname{var}\left(e_{i}^{s}\right)}{\sum_{i=1}^{N} \operatorname{var}\left(x_{i}^{s}\right)}
$$

where $s$ indexes the draw, and $S=1000$ is the number of replications. The properties of the estimates are judged by separately regressing $\widehat{F}_{1 t}$ and $\widehat{F}_{2 t}$ on a constant and the two dimensional $F_{t}^{0}$. The $R^{2}$ of the regressions indicate the coherence between $\widehat{F}_{k t}(k=1,2)$ and the space spanned by the true factors.

Of the three estimators considered, the PCA is the easiest to compute as there is no iteration involved. While ALS1 converges in a few iterations, ALS2 is computationally the most demanding. It is the only estimator that sometimes (albeit rarely) fails to converge. Furthermore, the ALS2 estimator cannot be implemented when $N>T$ and I mark these estimates with a '-'.

Table 1 reports results for the strong factor case with $\theta=1$. The top panel has time dependent but cross-sectionally uncorrelated idiosyncratic errors since $N_{c}=0$. With signAL above 0.5 , the three sets of estimated factors explain well over .95 of the variations in the true $F_{t}$. Assuming that 
$e_{i t}$ is cross-sectionally uncorrelated did not hurt the efficiency of PCA because the constraint is correct in this case.

The bottom panel of Table 1 allows the errors to be cross-sectionally correlated. As a consequence, the common component relative to the total variation in the data falls by as much as half. Notably, all factor estimates are less precise. One PCA factor tends to be more precisely estimated than the other. The discrepancy seems to increase as SIGNAL decreases. The two ALS estimators are much more even in this regard since $\widehat{F}_{1}$ and $\widehat{F}_{2}$ have similar predictive power of the factor space. Of the three estimators, ALS2 appears to be most unstable; it can be extremely good (such as when $(T, N)=(120,80)$ ) or extremely bad (such as when $T$ is increased to 240) holding $N_{c}$ fixed at $=8$. A factor that is precisely estimated by the PCA is not always precisely estimated by the ALS estimators and vice versa. When $(T, N)=(240,120), F_{1}^{0}$ is poorly estimated by the ALS estimators ( $R^{2}$ of .111 and .272 ) than by PCA (with $R^{2}$ of .524). However, the reverse is true of $F_{2}^{0}$, with $R^{2}$ of .824 and .739 for the ALS estimators, and only .355 for the PCA.

Table 2 considers weaker factor loadings with $\theta=0.5$. When the errors are not cross-sectionally correlated, SIGNAL in the top panel of Table 2 is reduced somewhat relative to Table 1 but the correlation is not enough to strongly affect the precision of the factor estimates. For example, when $(T, N)=(120,40), R^{2}$ is 0.960 when $\theta=1$ and is 0.865 when $\theta=.5$. When $(T, N)=(40,120), R^{2}$ goes from .987 to 0.94 . When the idiosyncratic errors are also cross-sectionally correlated, the drop in SIGNAL is much larger. The $R^{2}$ values in the second panel of Table 2 are one-third to one-quarter of those in Table 1. Weak loadings combined with cross-correlated errors drastically reduce the precision of the factor estimates irrespective of the method used. When $(T, N)=(240,120)$ which is not an unusual configuration of the data encountered in practice, signAL falls from .254 to .078 and the average $R^{2}$ drops from around .5 in Table 1 to less than .05 in Table 2! The difference is attributed to cross-correlated errors.

The results in Tables 1 and 2 are based on the assumption that $r$ is known. Bai and $\mathrm{Ng}(2002)$ show that the number of factors can be consistently estimated by minimizing $L_{P C A}$ subject to the constraint of parsimony. Specifically,

$$
\widehat{r}_{P C A}=\underset{k=k_{\min }, \ldots, k_{\max }}{\operatorname{argmin}} \log L_{P C A}(k)+k g(N, T)
$$

where $g(N, T) \rightarrow 0$ but $\min (N, T) g(N, T) \rightarrow \infty$. The ALS estimators are based on different objective functions. While their statistical properties are not known, Tables 1 and 2 find that the estimated ALS factors behave similarly to the PCA ones. I therefore let

$$
\widehat{r}_{A L S}=\underset{k=k_{\min }, \ldots, k_{\max }}{\operatorname{argmin}} \log L_{A L S}(k) / n T+k g(N, T) .
$$


In the simulations, I use

$$
g_{2}(N, T)=\frac{N+T}{N T} \log \min (N, T)
$$

noting that $N T /(N+T) \approx \min (N, T)^{-1}$. This corresponds to $I C_{2}$ recommended in Bai and $\mathrm{Ng}$ (2008). For the ALS estimators I also consider a heavier penalty

$$
g_{A}(N, T)=\frac{N+T}{N T} \log (N \cdot T)
$$

The simulation design is similar to Tables 1 and 2 . The criteria are evaluated for $k=0, \ldots, 6$. I only consider 500 replications because ALS2 is extremely time consuming to compute. The results are reported in Table 3 . When $\theta=1$ and the errors are cross-sectionally uncorrelated, the $I C_{2}$ almost always chooses the correct number of factors. The suitably penalized ALS objective functions also give the correct number of factors. The estimates of $r$ are imprecise when there is cross-section dependence in $e_{i t}$. The $g_{2}$ penalty often chooses the maximum number of factors whether PCA or ALS is used to estimate $F$, while the $g_{A}$ tends to select too few factors. The results are to be expected; the penalties developed in Bai and $\mathrm{Ng}$ (2002) are predicated on a strong factor structure with weakly correlated idiosyncratic errors. When those assumptions are violated, the penalties are no longer appropriate.

The AO criterion of Onatski (2010) is supposed to better handle situations when there is substantial correlation in the errors. As seen from the third panel of Table 3 , the AO criterion gives more precise estimates of $r$ when the factor loadings are weak. However, it tends to select zero factors when many of the idiosyncratic errors are cross-sectionally correlated. Onatski (2010) argues that his criterion selects the number of factors that can be consistently estimated. It is not surprising that the $\mathrm{AO}$ criterion selects fewer factors when the factor component is weak. But taking the argument at face value would suggest that when SIGNAL is below .3, none of the two factors can be consistently estimated by the PCA or the ALS. This seems at odds with the fact that the estimated factors still have substantial correlation with the true factors.

Two conclusions can be drawn from these simulations. First, the objective function used to obtain $\widehat{F}$ seems to make little difference as the PCA and ALS estimates are similar. Second, not constraining $\Omega$ to be diagonal (as in the PCA) or unnecessarily imposing the constraint (as in the ALS) also does not have much effect on $R^{2}$. In this regard, the results echo those of Doz, Giannone, and Reichlin (2007). If the strong factor assumptions hold true, there is little to choose between the estimators on the basis of the precise estimation of the factor space. Nonetheless, the PCA is computationally much less demanding.

Second, the precision of the factor estimates are strongly influenced by weak factor loadings. While signAL is not observed and it is not known if the factors are strong or weak in practice, two indicators can be useful. The first is $R^{2}$ which should increase with sIgnAL. A low $R^{2}$ in spite 
of using many factors would be a cause for concern. The second is the discrepancy between the number of factors selected by $I C_{2}$ and $\mathrm{AO}$. The two estimates should not be far apart when the factor structure is strong. When the $\widehat{r}_{\mathrm{s}}$ are very different, the strong factor assumptions may be questionable.

\subsection{Mixed Data}

Two designs of categorical data are considered. In the first case, the ordinal data $x$ consists of answers by $N$ respondents (such as professional forecasters) to the same question (such as whether they expect inflation to go up, down, or stay the same) over time $T$ periods. In the second case, $x$ consists of $J$ responses (such as on income and health status) for each of the $N$ units (such as households). In these experiments, PCA is used to estimate $\widehat{r}$ factors in (i) the continuous data $X$ as if it were observed, (ii) the categorical data $x$, (iii) the adjacency matrix $G$, and (where appropriate) (iv) the quantified data $Z$. The number of factors is determined by the criterion in Bai and $\mathrm{Ng}$ (2002) with penalty $g_{2}$ or the AO test of Onatski (2010). I begin with the first case when all data are ordinal.

a) PCA of $X, x$ : and $G$ Data on one variable $X$ are generated for $N$ units for $T$ time periods. The $J-1$ thresholds are evenly spaced and are generated using NORMinv(.05:J-1:.95). The data matrix is first standardized columnwise and then categorized into $x$ which has $J$ groups.

The results are given in Table 4 . Columns 3 and 4 show that if $X$ was observed, the number of factors would be precisely estimated. As seen from column 5 and $6, \widehat{r}$ remains fairly precisely estimated when the categorical data $x$ are used instead of $X$. However, the estimated number of factors in the adjacency matrix $G$ is less stable. There are too few factors when the sample size is small but too many factors when $N$ and $T$ are large. ${ }^{11}$

Turning to an assessment of the estimated factor space, $R_{X}^{2}$ indicates the average $R^{2}$ when $\widehat{r}$ principal components are estimated from $X$ where the $\widehat{r}$ is determined by penalty $g_{2}$. The interpretation is similar for $R_{x}^{2}$ and $R_{G}^{2}$. Evidently, $\widehat{F}$ precisely estimates $F$ when $X$ was available for analysis. The $R^{2}$ s are slightly lower if the factors are estimated from $x$ but the difference is quite small. The average $R^{2}$ remains well over .95 . However, the principal components of the adjacency matrix $G$ are less informative about the true factors. When the sample size is small and $\widehat{r}$ underestimates $r, R_{G}^{2}$ can be much lower than $R_{x}^{2}$. For example, when $r=2, R_{x}^{2}$ is .916 when $(T, N)=(100,20)$, but $R_{G}^{2}$ is only .368 . The situation improves when the sample size increases as estimating more factors in $G$ compensates for the information loss in the indicator variables.

\footnotetext{
${ }^{11}$ In an earlier version of the paper when $x$ and $G$ were not demeaned, PCA estimated one more factor in both $x$ and $G$.
} 
However, even with large $\widehat{r}$, the principal components of $G$ remain less informative about $F^{0}$ than the principal components of $x$. When $(T, N)=(200,100), R_{x}^{2}$ is .956 while $R_{G}^{2}$ is .849, even though on average, $\widehat{r}=3>r=2$ factors are found in $G$.

b) PCA of $X, x, G$ and $Z$ Data for $J$ variables for each of the $N$ units are generated as:

$$
X_{i j}=\Lambda_{i,:}^{0} F_{t}^{0}+e_{i j}
$$

where $e_{i j} \sim N\left(0, \sigma^{2}\right), \Lambda_{i,:}^{0}$ is a $1 \times r$ vector of standard normal variates, $F_{t}^{0} \sim N\left(0, I_{r}\right)$. The factor loadings are $N\left(0, \theta^{2}\right)$. The $J$ continuous variables are categorized using unevenly spaced thresholds as summarized in the bottom of Table 5. The total number of categories (also the dimension of $G$ ) is denoted $n_{G}$.

The results for the case of strong loadings $(\theta=1)$ are in the top panel of Table 5. As seen from columns 5 to 8 , the number of factors is overestimated whenever $J$ is small even if $X$ was observed; the average $R^{2}$ is .75 when $J$ is 5 but increases to .941 when $J=30$. This just shows that principal components can precisely estimate the factor space only when $J$ is reasonably large. There is undoubtedly a loss of precision when $X$ is not observed. The principal components of $G$ yield low $R^{2}$ for small values of $J$. Apparently, $G$ contains less information about $F$ than $Z$. While the number of factors found in the discrete data $x$ exceeds $r$, the $\widehat{r}$ principal components of $x$ give $R^{2}$ s close to those based on $Z$. In other words, with enough estimated factors, the factor space can be as precisely estimated from discrete data than as from the quantified data.

The lower panel of Table 5 are results for the case of weak loadings with $\theta=.5$. Now $R_{Z}^{2}$ and $R_{G}^{2}$ are much reduced as the corresponding number of factors found in $Z$ and $G$ tends to be below the true value of two. This suggests that when the factor structure is already weak, discretization further weakens the information about the common factors in $x$ which makes it difficult to recover $F$ from quantified data or additional transformations of $x$. In such a case, the principal components of the raw categorical data $x$ give the most precise factor estimates and they are easiest to construct.

The final set of simulations consider mixed continuous, nominal and ordinal data. The number of continuous variables is always 20 , the number of nominal variables $J_{\text {nominal }}$ is either 5 or 10 , and the number of ordinal variables $J_{\text {ordinal }}$ is 5,10 , or $20 .{ }^{12}$ The results are given in Table 6 . The number of factors is correctly estimated from the continuous data $X$, the quantified data $Z$, or the discrete data $x$ but are overestimated from the dummy variable matrix $G$. The factor space is precisely estimated using $X, Z$ or $x$ but not $G$. The results are similar to those for purely ordinal data.

\footnotetext{
${ }^{12}$ The FACTALS has convergence problems when $N$ is 100 and the dimension of $G$ is large.
} 


\section{Conclusion}

This paper reviews and explores various matrix decomposition based methods for estimating the common factors in mixed data. A few conclusions can be drawn. First, if all data are continuous and $T$ and $N$ are large, ALS estimators have no significant advantage over PCA which is much simpler to construct. Second, with mixed data, the principal components of $G$ give the least precise estimates of the factor space. Third, FACTALS provides a way to quantify the data consistent with a factor structure. But while the factor space can be precisely obtained from $Z$ when the factor structure is strong, they are no more precise than analyzing $x$ directly, and it is not robust when the factor structure in $X$ is weak. Fourth, the observed categorical data $x$ can be used to estimate the factor space quite precisely though additional factors may be necessary to compensate for the information that is lost from data discretization. It should be emphasized that different conclusions might emerge if criterion other than the factor space is used. Furthermore, the Monte Carlo exercise is quite limited in scope. Nonetheless, the findings are encouraging and merit further investigation.

I consider FACTALS because it constructs quantified data $Z$ consistent with a factor structure which allows me to consider a large dimensional factor analysis treating $Z$ as data. However, as pointed out earlier, the sample correlations of transformed data are not the same as the raw data. Nothing ensures that $Z$ will necessarily recover $X$ from $x$. Thus, an important caveat of nonmetrical factor analysis is that $Z$ may not be unique even when its second moments are consistent with the factor structure implied by the data $X$. Buja (1990) warns that the transformed data may identify spurious structures because singular values are sensitive to small changes in the data. A better understanding of these issues is also necessary. 


\section{Appendix}

This appendix elaborates on how nominal and ordinal data are optimally scaled. It will be seen that the steps rely on matrix arguments presented in Section 3.

Step 3b: Nominal Variables Observe first that $Z_{:,-j}^{\prime} G_{j} y_{j}=Z_{:,-j}^{\prime} Z_{:, j}$ defines the vector of 'sample' cross correlations, while $\widehat{R}_{Z,-j}$ is the model implied analog. The $j$-entry is omitted since it is one by construction in both the sample and the model. We put sample in quotes because $Z$ are quantified variables and not data.

Let $M=I_{T}-11^{\prime} / T$ be the idempotent matrix that demeans the data. Constraining $Z$ to be mean zero columnwise is equivalent to imposing the condition $M G_{j} y_{j}=G_{j} y_{j}$ for every $j$ with $Z_{:,-j}^{\prime} G_{j} y_{j}=Z_{:,-j}^{\prime} M G_{j} y_{j}$. Let $B$ be an orthonormal bases for $M G_{j} y_{j}$. Then $M G_{j} y_{j}=B \tau$ for some $\tau$. Instead of minimizing $\left\|Z_{:,-j}^{\prime} G_{j} y_{j}-\widehat{R}_{Z,:,-j}\right\|^{2}$ over $y_{j}$ subject to the constraint $y_{j}^{\prime} G_{j}^{\prime} G_{j} y_{j}=$ $y_{j}^{\prime} G_{j}^{\prime} M G_{j} y_{j}$, the problem is now to minimize

$$
\left\|Z_{-j,:}^{\prime} B \tau-\widehat{R}_{Z,:,-j}\right\|^{2}
$$

over $\tau$ subject to the constraint that $\tau^{\prime} B^{\prime} B \tau=\tau^{\prime} \tau=1$. This is an oblique Mosier's Procrustes problem whose solution, denoted $\tau_{0}$, is given in Cliff (1966) and ten Berge and Nevels (1997). Given $\tau_{0}, y_{j}$ can be solved from $M G_{j} y_{j}=B \tau_{0}$. By least squares argument,

$$
y_{j}^{0}=\left(G_{j}^{\prime} G_{j}\right)^{-1} G_{j}^{\prime} B \tau_{0}
$$

It remains to explain the oblique Procrustes problem. Recall that the orthogonal Procrustes problem looks for a $m \times k$ transformation matrix $B$ to minimize $\|\Lambda-A B\|^{2}$ subject to $B^{\prime} B=I$. The oblique Procrustes problem imposes the constraint $\operatorname{diag}\left(B^{\prime} B\right)=I$. Since the constraint is now a diagonal matrix, each column of $B$ can be solved separately. Let $\beta$ be a vector from $B$, and $\lambda$ be a column of $\Lambda$. The objective is now to find $\beta$ to minimize $(\lambda-A \beta)^{\prime}(\lambda-A \beta)$. For $U$ such that $U U^{\prime}=U^{\prime} U=I$ and $A^{\prime} A=U C U^{\prime}$, the problem can be rewritten as

$$
(\lambda-A \beta)^{\prime}(\lambda-A \beta)=(\lambda-A U \beta)^{\prime}(\lambda-A U \beta)
$$

By letting $w=U^{\prime} \beta$ and $x=U^{\prime} A \lambda$, the problem is equivalent to finding a vector $w$ to minimize

$$
\lambda^{\prime} \lambda-2 x^{\prime} w+w^{\prime} C w \quad \text { subject to } w^{\prime} w=1 .
$$

The objective function and the constraint are both in quadratic form. The (non-linear) solution depends on $q$, the multiplicity of the smallest latent root of $A^{\prime} A$. In the simulations, I use the algorithm of ten Berge and Nevels (1997). In the present problem, $\lambda=\widehat{R}_{Z,:-j}$ and $A=Z_{:,-j}^{\prime} B$. 
Step 3c: Ordinal Variables When $X_{:, j}$ is ordinal, we minimize a function that majorizes $f\left(y_{j}\right)$ and whose solution is easier to find. As shown in Kiers (1990), this is accomplished by viewing $f$ as a function of $q=G_{j} y_{j}$. The function of interest (for given $q^{0}$ )

$$
f(q)=\left\|Z_{:,-j}^{\prime} q-\widehat{R}_{Z,:,-j}\right\|=\widehat{R}_{Z,:,-j}^{\prime} \widehat{R}_{Z,:,-j}-2 \widehat{R}_{Z,:,-j}^{\prime} Z_{:,-j}^{\prime} q+\operatorname{trace}\left(Z_{:,-j} Z_{:,-j}^{\prime} q q^{\prime}\right)
$$

is majorized by

$$
g(q)=c_{1}+a\left(\left\|q^{0}-(2 a)^{-1}\left(-2 Z_{:,-j} \widehat{R}_{Z,:,-j}+2 Z_{:,-j} Z_{:,-j}^{\prime} q^{0}\right)-q\right\|^{2}+c_{2}\right)
$$

where $c_{1}$ and $c_{2}$ are constants for $q$, and $a$ is the first eigenvalue of $Z_{:,-j} Z_{:,-j}^{\prime}$. Re-expressing $q_{j}$ in terms of $G_{j} y_{j}$, we can maximize

$$
\begin{aligned}
h\left(y_{j}\right) & =\left\|G_{j} y_{j}^{0}-(2 a)^{-1}\left(-2 Z_{:,-j} \widehat{R}_{Z,:,-j}+2 Z_{:,-j} Z_{:,-j}^{\prime} G_{j} G_{j}^{0}\right)-G_{j} y_{j}\right\|^{2} \\
& =\left\|\left(G_{j} y_{j}^{0}+a^{-1} Z_{:,-j} \widehat{R}_{Z,:,-j}-a^{-1} Z_{:,-j} Z_{:,-j}^{\prime} G_{j} y_{j}^{0}\right)-G_{j} y_{j}\right\|^{2} \\
& =\left\|z-G_{j} y_{j}\right\|^{2}
\end{aligned}
$$

subject to the constraints that $G_{j} y_{j}$ is centered and $y_{j}^{\prime} G_{j}^{\prime} G_{j} y_{j}=1$. This is now a normalized monotone regression problem. ${ }^{13}$

\footnotetext{
${ }^{13}$ Given weights $w_{1}, \ldots, w_{T}$ and real numbers $x_{1}, \ldots, x_{T}$, the monotone (isotonic) regression problem finds $\widehat{x}_{1}, \ldots \widehat{x}_{T}$ to minimize $S(y)=\sum_{t=1}^{T} w_{t}\left(x_{t}-y_{t}\right)^{2}$ subject to the monotonicity condition $t \preceq k$ implies $y_{t} \leq y_{k}$ where $\preceq$ is a partial ordering on the index set $[1, \ldots T]$. An up-and-down-block algorithm is given in Kruskal (1964). See also de Leeuw (2005).
} 
Table 1: $R^{2}$ from Regressions of $\widehat{F}_{j}$ on $F: \theta=1$.

\begin{tabular}{|c|c|c|c|c|c|c|c|c|c|}
\hline \multirow[t]{2}{*}{$T$} & \multirow[t]{2}{*}{$N$} & \multirow[t]{2}{*}{$N_{c}$} & \multirow[t]{2}{*}{ SIGNAL } & $\widehat{F}_{1}$ & $\widehat{F}_{2}$ & $\widehat{F}_{1}$ & $\widehat{F}_{2}$ & $\widehat{F}_{2}$ & $\widehat{F}_{2}$ \\
\hline & & & & \multicolumn{2}{|c|}{$\mathrm{PCA}$} & \multicolumn{2}{|c|}{ ALS I } & \multicolumn{2}{|c|}{ ALS II } \\
\hline 120 & 40 & 0 & 0.520 & 0.960 & 0.961 & 0.941 & 0.940 & 0.960 & 0.960 \\
\hline 120 & 80 & 0 & 0.548 & 0.981 & 0.980 & 0.971 & 0.971 & 0.979 & 0.970 \\
\hline 120 & 120 & 0 & 0.598 & 0.987 & 0.985 & 0.986 & 0.986 & - & - \\
\hline 120 & 240 & 0 & 0.599 & 0.994 & 0.993 & 0.994 & 0.994 & - & - \\
\hline 240 & 40 & 0 & 0.579 & 0.975 & 0.961 & 0.944 & 0.953 & 0.962 & 0.974 \\
\hline 240 & 80 & 0 & 0.593 & 0.984 & 0.978 & 0.978 & 0.969 & 0.985 & 0.979 \\
\hline 240 & 120 & 0 & 0.592 & 0.988 & 0.986 & 0.981 & 0.981 & 0.988 & 0.987 \\
\hline 480 & 40 & 0 & 0.564 & 0.964 & 0.948 & 0.927 & 0.938 & 0.947 & 0.964 \\
\hline 480 & 80 & 0 & 0.582 & 0.980 & 0.977 & 0.968 & 0.966 & 0.977 & 0.981 \\
\hline 480 & 120 & 0 & 0.588 & 0.989 & 0.985 & 0.979 & 0.982 & 0.988 & 0.985 \\
\hline 480 & 240 & 0 & 0.607 & 0.994 & 0.001 & 0.991 & 0.992 & 0.993 & 0.994 \\
\hline 40 & 120 & 0 & 0.610 & 0.987 & 0.984 & 0.987 & 0.987 & - & - \\
\hline 80 & 120 & 0 & 0.601 & 0.987 & 0.984 & 0.987 & 0.987 & - & - \\
\hline 120 & 40 & 4 & 0.432 & 0.866 & 0.918 & 0.896 & 0.852 & 0.821 & 0.921 \\
\hline 120 & 80 & 8 & 0.302 & 0.797 & 0.878 & 0.780 & 0.880 & 0.905 & 0.827 \\
\hline 120 & 120 & 12 & 0.268 & 0.520 & 0.492 & 0.540 & 0.532 & - & - \\
\hline 120 & 240 & 24 & 0.173 & 0.104 & 0.131 & 0.124 & 0.137 & - & - \\
\hline 240 & 40 & 4 & 0.487 & 0.972 & 0.924 & 0.933 & 0.923 & 0.955 & 0.970 \\
\hline 240 & 80 & 8 & 0.330 & 0.928 & 0.917 & 0.897 & 0.900 & 0.803 & 0.142 \\
\hline 240 & 120 & 12 & 0.254 & 0.524 & 0.355 & 0.111 & 0.824 & 0.272 & 0.739 \\
\hline 480 & 40 & 4 & 0.466 & 0.922 & 0.901 & 0.896 & 0.893 & 0.930 & 0.874 \\
\hline 480 & 80 & 8 & 0.320 & 0.689 & 0.826 & 0.718 & 0.824 & 0.660 & 0.691 \\
\hline 480 & 120 & 12 & 0.260 & 0.664 & 0.446 & 0.832 & 0.309 & 0.350 & 0.781 \\
\hline 480 & 240 & 24 & 0.182 & 0.003 & 0.044 & 0.019 & 0.032 & 0.012 & 0.467 \\
\hline 40 & 120 & 12 & 0.277 & 0.518 & 0.549 & 0.548 & 0.560 & - & - \\
\hline 80 & 120 & 12 & 0.270 & 0.494 & 0.515 & 0.531 & 0.539 & - & - \\
\hline
\end{tabular}


Table 2: $R^{2}$ from Regressions of $\widehat{F}_{j}$ on $F: \theta=.5$.

\begin{tabular}{llll|ll|lll|ll}
$T$ & $N$ & $N_{c}$ & SIGNAL & $\widehat{F}_{1}$ & \multicolumn{2}{|c|}{$\widehat{F}_{2}$} & $\widehat{F}_{1}$ & $\widehat{F}_{2}$ & $\widehat{F}_{1}$ & \multicolumn{1}{|c}{$\widehat{F}_{2}$} \\
\hline & & & & \multicolumn{2}{|c|}{ PCA } & \multicolumn{2}{|c}{ ALS I } & \multicolumn{2}{c}{ ALS II } \\
\hline \hline 120 & 40 & 0 & 0.217 & 0.865 & 0.857 & 0.776 & 0.730 & 0.846 & 0.866 \\
120 & 80 & 0 & 0.235 & 0.928 & 0.934 & 0.910 & 0.896 & 0.909 & 0.891 \\
120 & 120 & 0 & 0.272 & 0.956 & 0.945 & 0.950 & 0.950 & - & - \\
120 & 240 & 0 & 0.272 & 0.977 & 0.973 & 0.975 & 0.975 & - & - \\
240 & 40 & 0 & 0.259 & 0.918 & 0.879 & 0.771 & 0.845 & 0.875 & 0.916 \\
240 & 80 & 0 & 0.267 & 0.951 & 0.922 & 0.904 & 0.889 & 0.951 & 0.918 \\
240 & 120 & 0 & 0.266 & 0.961 & 0.953 & 0.919 & 0.932 & 0.960 & 0.955 \\
480 & 40 & 0 & 0.244 & 0.889 & 0.831 & 0.754 & 0.769 & 0.835 & 0.878 \\
480 & 80 & 0 & 0.256 & 0.930 & 0.928 & 0.872 & 0.878 & 0.931 & 0.927 \\
480 & 120 & 0 & 0.264 & 0.962 & 0.952 & 0.920 & 0.930 & 0.961 & 0.954 \\
480 & 240 & 0 & 0.278 & 0.979 & 0.979 & 0.969 & 0.963 & 0.968 & 0.967 \\
40 & 120 & 0 & 0.281 & 0.943 & 0.929 & 0.937 & 0.936 & - & - \\
80 & 120 & 0 & 0.274 & 0.954 & 0.942 & 0.948 & 0.948 & - & - \\
\hline 120 & 40 & 4 & 0.161 & 0.326 & 0.451 & 0.377 & 0.332 & 0.410 & 0.176 \\
120 & 80 & 8 & 0.098 & 0.195 & 0.023 & 0.037 & 0.192 & 0.209 & 0.032 \\
120 & 120 & 12 & 0.084 & 0.047 & 0.052 & 0.050 & 0.055 & - & - \\
120 & 240 & 24 & 0.050 & 0.032 & 0.035 & 0.033 & 0.036 & - & - \\
240 & 40 & 4 & 0.192 & 0.445 & 0.387 & 0.449 & 0.371 & 0.647 & 0.748 \\
240 & 80 & 8 & 0.110 & 0.009 & 0.022 & 0.021 & 0.026 & 0.030 & 0.011 \\
240 & 120 & 12 & 0.078 & 0.007 & 0.036 & 0.003 & 0.040 & 0.011 & 0.041 \\
480 & 40 & 4 & 0.179 & 0.364 & 0.266 & 0.190 & 0.418 & 0.274 & 0.196 \\
480 & 80 & 8 & 0.105 & 0.088 & 0.033 & 0.010 & 0.113 & 0.026 & 0.047 \\
480 & 120 & 12 & 0.081 & 0.012 & 0.007 & 0.008 & 0.013 & 0.001 & 0.045 \\
480 & 240 & 24 & 0.053 & 0.002 & 0.003 & 0.003 & 0.002 & 0.002 & 0.014 \\
40 & 120 & 12 & 0.088 & 0.118 & 0.121 & 0.126 & 0.125 & - & - \\
80 & 120 & 12 & 0.085 & 0.064 & 0.068 & 0.071 & 0.070 & - & - \\
\hline
\end{tabular}


Table 3: Estimates of $r=2$ Using Continuous Data

\begin{tabular}{|c|c|c|c|c|c|c|c|c|c|}
\hline$T$ & $N$ & $N_{c}$ & SIGNAL & $g_{2}$ & $g_{2}$ & $g_{A}$ & $g_{2}$ & $g_{A}$ & $A O$ \\
\hline & & $=1$ & & $\mathrm{PCA}$ & & & & & PCA \\
\hline 120 & 40 & 0 & 0.497 & 2.002 & 2.006 & 2.000 & 1.998 & 1.010 & 2.040 \\
\hline 120 & 80 & 0 & 0.495 & 2.000 & 2.000 & 2.000 & 2.142 & 1.634 & 2.072 \\
\hline 120 & 240 & 0 & 0.495 & 2.000 & 2.000 & 2.000 & 3.378 & 1.746 & 2.006 \\
\hline 240 & 40 & 0 & 0.490 & 2.000 & 2.026 & 2.000 & 1.996 & 1.022 & 2.020 \\
\hline 240 & 80 & 0 & 0.489 & 2.000 & 2.000 & 2.000 & 2.006 & 1.932 & 2.030 \\
\hline 240 & 120 & 0 & 0.491 & 2.000 & 2.000 & 2.000 & 2.002 & 1.998 & 2.022 \\
\hline 480 & 40 & 0 & 0.486 & 2.000 & 2.026 & 2.000 & 2.004 & 1.010 & 2.010 \\
\hline 480 & 80 & 0 & 0.488 & 2.000 & 2.000 & 2.000 & 2.012 & 1.966 & 2.026 \\
\hline 480 & 120 & 0 & 0.487 & 2.000 & 2.000 & 2.000 & 2.002 & 1.998 & 2.020 \\
\hline 480 & 240 & 0 & 0.488 & 2.000 & 2.000 & 2.000 & 2.004 & 2.004 & 2.006 \\
\hline 120 & 40 & 4 & 0.423 & 6.000 & 5.732 & 1.448 & 1.912 & 1.000 & 1.838 \\
\hline 120 & 80 & 8 & 0.300 & 6.000 & 5.998 & 1.008 & 4.782 & 1.002 & 0.276 \\
\hline 120 & 240 & 24 & 0.165 & 6.000 & 6.000 & 1.002 & 5.242 & 1.092 & 0.010 \\
\hline 240 & 40 & 4 & 0.418 & 6.000 & 6.000 & 1.622 & 1.994 & 1.000 & 1.908 \\
\hline 240 & 80 & 8 & 0.300 & 6.000 & 6.000 & 1.112 & 5.860 & 1.002 & 0.184 \\
\hline 240 & 120 & 12 & 0.239 & 6.000 & 6.000 & 4.600 & 5.966 & 1.000 & 0.004 \\
\hline 480 & 40 & 4 & 0.415 & 6.000 & 6.000 & 1.712 & 2.022 & 1.000 & 1.946 \\
\hline 480 & 80 & 8 & 0.300 & 6.000 & 6.000 & 1.970 & 5.920 & 1.002 & 0.132 \\
\hline 480 & 120 & 12 & 0.236 & 6.000 & 6.000 & 6.000 & 5.986 & 1.000 & 0.000 \\
\hline 480 & 240 & 24 & 0.162 & 6.000 & 6.000 & 6.000 & 5.992 & 5.922 & 0.000 \\
\hline & & & $\theta=.5$ & & & & & & \\
\hline 120 & 40 & 0 & 0.086 & 0.004 & 1.010 & 1.000 & 1.000 & 1.000 & 0.488 \\
\hline 120 & 80 & 0 & 0.085 & 0.000 & 1.000 & 1.000 & 1.300 & 1.000 & 0.918 \\
\hline 120 & 240 & 0 & 0.086 & 0.658 & 1.000 & 1.000 & 1.124 & 1.000 & 1.960 \\
\hline 240 & 40 & 0 & 0.084 & 0.006 & 1.384 & 1.000 & 1.000 & 1.000 & 1.164 \\
\hline 240 & 80 & 0 & 0.084 & 0.096 & 1.614 & 1.000 & 1.000 & 1.000 & 1.930 \\
\hline 240 & 120 & 0 & 0.084 & 0.606 & 1.682 & 1.000 & 1.002 & 1.000 & 2.016 \\
\hline 480 & 40 & 0 & 0.083 & 0.008 & 1.940 & 1.006 & 1.000 & 1.000 & 1.888 \\
\hline 480 & 80 & 0 & 0.083 & 0.396 & 2.000 & 1.064 & 1.000 & 1.000 & 2.014 \\
\hline 480 & 120 & 0 & 0.083 & 1.452 & 2.000 & 1.178 & 1.000 & 1.000 & 2.020 \\
\hline 480 & 240 & 0 & 0.083 & 2.000 & 2.000 & 1.440 & 1.008 & 1.000 & 2.008 \\
\hline & & & $\theta=.2$ & & & & & & \\
\hline 120 & 40 & 0 & 0.244 & 1.938 & 2.004 & 1.280 & 1.010 & 1.000 & 2.034 \\
\hline 120 & 80 & 0 & 0.242 & 2.000 & 2.000 & 1.324 & 1.618 & 1.024 & 2.072 \\
\hline 120 & 240 & 0 & 0.243 & 2.000 & 2.000 & 1.092 & 1.406 & 1.022 & 2.004 \\
\hline 240 & 40 & 0 & 0.239 & 1.982 & 2.004 & 1.924 & 1.064 & 1.000 & 2.066 \\
\hline 240 & 80 & 0 & 0.238 & 2.000 & 2.000 & 2.000 & 1.662 & 1.002 & 2.022 \\
\hline 240 & 120 & 0 & 0.239 & 2.000 & 2.000 & 2.000 & 1.958 & 1.052 & 2.012 \\
\hline 480 & 40 & 0 & 0.236 & 1.996 & 2.002 & 2.000 & 1.094 & 1.000 & 2.038 \\
\hline 480 & 80 & 0 & 0.237 & 2.000 & 2.000 & 2.000 & 1.874 & 1.000 & 2.042 \\
\hline 480 & 120 & 0 & 0.237 & 2.000 & 2.000 & 2.000 & 1.962 & 1.078 & 2.044 \\
\hline 480 & 240 & 0 & 0.237 & 2.000 & 2.000 & 2.000 & 2.032 & 1.576 & 2.008 \\
\hline
\end{tabular}


Table 4: Estimates of $r$ from Ordinal Data

\begin{tabular}{|c|c|c|c|c|c|c|c|c|c|c|}
\hline & & \multicolumn{6}{|c|}{ Number of Factors } & \multicolumn{3}{|c|}{$R^{2}$} \\
\hline$T$ & $N$ & $I C_{X}$ & $A O_{X}$ & $I C_{x}$ & $A O_{x}$ & $I C_{G}$ & $A O_{G}$ & $R_{X}^{2}$ & $R_{x}^{2}$ & $R_{G}^{2}$ \\
\hline \multicolumn{11}{|c|}{$r=1$} \\
\hline 50 & 20 & 1.050 & 1.014 & 1.000 & 1.014 & 0.854 & 0.914 & 0.959 & 0.927 & 0.557 \\
\hline 100 & 20 & 1.052 & 1.006 & 1.000 & 1.026 & 1.140 & 1.156 & 0.959 & 0.929 & 0.676 \\
\hline 200 & 20 & 1.060 & 1.008 & 1.000 & 1.024 & 1.342 & 1.310 & 0.959 & 0.928 & 0.687 \\
\hline 50 & 50 & 1.000 & 1.010 & 1.000 & 1.026 & 1.136 & 1.606 & 0.984 & 0.953 & 0.777 \\
\hline 100 & 50 & 1.000 & 1.010 & 1.000 & 1.018 & 1.502 & 1.962 & 0.984 & 0.955 & 0.791 \\
\hline 200 & 50 & 1.000 & 1.006 & 1.000 & 1.010 & 1.890 & 2.144 & 0.984 & 0.954 & 0.796 \\
\hline 50 & 100 & 1.000 & 1.004 & 1.000 & 1.018 & 1.244 & 2.016 & 0.992 & 0.963 & 0.826 \\
\hline 100 & 100 & 1.000 & 1.010 & 1.000 & 1.006 & 1.872 & 2.368 & 0.992 & 0.962 & 0.830 \\
\hline 200 & 100 & 1.000 & 1.016 & 1.000 & 1.058 & 2.006 & 2.864 & 0.992 & 0.962 & 0.831 \\
\hline \multicolumn{11}{|c|}{$r=2$} \\
\hline 50 & 20 & 2.714 & 2.024 & 2.000 & 2.028 & 0.324 & 0.524 & 0.958 & 0.919 & 0.119 \\
\hline 100 & 20 & 3.302 & 2.032 & 2.000 & 2.020 & 1.126 & 1.004 & 0.960 & 0.916 & 0.368 \\
\hline 200 & 20 & 3.424 & 2.014 & 2.000 & 2.010 & 1.916 & 1.458 & 0.959 & 0.917 & 0.577 \\
\hline 50 & 50 & 2.000 & 2.034 & 2.000 & 2.016 & 1.236 & 1.502 & 0.984 & 0.949 & 0.488 \\
\hline 100 & 50 & 2.000 & 2.016 & 2.000 & 2.024 & 2.090 & 2.344 & 0.984 & 0.947 & 0.750 \\
\hline 200 & 50 & 2.000 & 2.010 & 2.000 & 2.016 & 2.784 & 2.902 & 0.984 & 0.947 & 0.797 \\
\hline 50 & 100 & 2.000 & 2.014 & 2.000 & 2.022 & 1.742 & 2.138 & 0.992 & 0.958 & 0.708 \\
\hline 100 & 100 & 2.000 & 2.004 & 2.000 & 2.062 & 2.710 & 2.630 & 0.992 & 0.957 & 0.841 \\
\hline 200 & 100 & 2.000 & 2.018 & 2.000 & 2.184 & 3.092 & 2.842 & 0.992 & 0.956 & 0.849 \\
\hline \multicolumn{11}{|c|}{$r=3$} \\
\hline 50 & 20 & 4.212 & 3.012 & 2.998 & 3.020 & 0.082 & 0.398 & 0.960 & 0.908 & 0.022 \\
\hline 100 & 20 & 4.450 & 3.002 & 3.000 & 3.018 & 0.330 & 0.518 & 0.960 & 0.906 & 0.085 \\
\hline 200 & 20 & 4.478 & 3.002 & 3.000 & 3.004 & 1.040 & 0.852 & 0.960 & 0.906 & 0.249 \\
\hline 50 & 50 & 3.006 & 3.032 & 3.000 & 3.012 & 0.336 & 0.716 & 0.984 & 0.944 & 0.103 \\
\hline 100 & 50 & 3.028 & 3.012 & 3.000 & 3.030 & 1.358 & 1.704 & 0.984 & 0.943 & 0.382 \\
\hline 200 & 50 & 3.138 & 3.014 & 3.000 & 3.036 & 2.756 & 2.776 & 0.984 & 0.942 & 0.686 \\
\hline 50 & 100 & 3.000 & 3.006 & 3.000 & 3.032 & 0.760 & 1.290 & 0.992 & 0.955 & 0.243 \\
\hline 100 & 100 & 3.000 & 3.014 & 3.000 & 3.018 & 2.750 & 2.106 & 0.992 & 0.954 & 0.751 \\
\hline 200 & 100 & 3.000 & 3.008 & 3.000 & 3.054 & 3.930 & 2.756 & 0.992 & 0.953 & 0.868 \\
\hline
\end{tabular}

For $y=X$ (latent continuous data), $x$ (categorical data), and $G$ (adjacency matrix of indicators), $I C_{y}$ denotes the number of factors selected by the Bai and $\mathrm{Ng}(2002)$ criterion with penalty $g_{2}=\frac{N+T}{N T} \log \min (N, T)$ when the principal components are constructed from data $y . A O_{y}$ denotes factors determined using the criterion of Onatski (2010). The columns $R^{2} y$ denote the average $R^{2}$ when each of the $\widehat{r}$ factors estimated from $y$ are regressed on the true factors. 
Table 5: Ordinal Data, $r=2$

\begin{tabular}{|c|c|c|c|c|c|c|c|c|c|c|}
\hline$N$ & $J$ & $n_{G}$ & $\widehat{r}_{X}$ & $\widehat{r}_{G}$ & $\widehat{r}_{Z}$ & $\widehat{r}_{x}$ & $R_{X}^{2}$ & $R_{G}^{2}$ & $R_{Z}^{2}$ & $R_{x}^{2}$ \\
\hline \multicolumn{11}{|c|}{$\theta=1$} \\
\hline 100 & 5 & 18 & 4.000 & 3.513 & 4.000 & 4.000 & 0.742 & 0.475 & 0.609 & 0.630 \\
\hline 100 & 10 & 33 & 3.828 & 2.648 & 3.013 & 3.993 & 0.798 & 0.497 & 0.650 & 0.686 \\
\hline 100 & 15 & 50 & 3.332 & 2.346 & 2.646 & 3.824 & 0.832 & 0.544 & 0.690 & 0.723 \\
\hline 200 & 5 & 18 & 4.000 & 3.777 & 4.000 & 4.000 & 0.734 & 0.481 & 0.599 & 0.616 \\
\hline 200 & 10 & 33 & 3.806 & 2.971 & 3.051 & 3.994 & 0.793 & 0.527 & 0.652 & 0.675 \\
\hline 200 & 15 & 50 & 3.314 & 2.677 & 2.688 & 3.847 & 0.830 & 0.590 & 0.701 & 0.715 \\
\hline 100 & 20 & 60 & 2.025 & 1.945 & 1.898 & 3.569 & 0.928 & 0.744 & 0.783 & 0.811 \\
\hline 100 & 30 & 80 & 2.013 & 2.001 & 1.848 & 3.693 & 0.941 & 0.778 & 0.770 & 0.821 \\
\hline 200 & 20 & 60 & 2.015 & 2.209 & 1.966 & 3.695 & 0.928 & 0.787 & 0.817 & 0.811 \\
\hline 200 & 30 & 80 & 2.007 & 2.305 & 1.958 & 3.814 & 0.941 & 0.811 & 0.819 & 0.818 \\
\hline \multicolumn{11}{|c|}{$\theta=.5$} \\
\hline 100 & 5 & 18 & 4.000 & 2.212 & 4.000 & 4.000 & 0.487 & 0.219 & 0.384 & 0.400 \\
\hline 100 & 10 & 33 & 2.644 & 1.289 & 2.237 & 4.000 & 0.462 & 0.164 & 0.269 & 0.446 \\
\hline 100 & 15 & 50 & 1.433 & 0.331 & 0.436 & 2.096 & 0.562 & 0.115 & 0.155 & 0.458 \\
\hline 200 & 5 & 18 & 4.000 & 2.594 & 4.000 & 4.000 & 0.469 & 0.234 & 0.369 & 0.377 \\
\hline 200 & 10 & 33 & 2.663 & 1.600 & 2.228 & 4.000 & 0.472 & 0.205 & 0.270 & 0.430 \\
\hline 200 & 15 & 50 & 1.556 & 0.735 & 0.431 & 2.159 & 0.602 & 0.240 & 0.155 & 0.470 \\
\hline 100 & 20 & 60 & 1.641 & 0.423 & 0.453 & 2.710 & 0.669 & 0.155 & 0.165 & 0.529 \\
\hline 100 & 30 & 80 & 1.769 & 0.488 & 0.412 & 3.159 & 0.744 & 0.184 & 0.153 & 0.562 \\
\hline 200 & 20 & 60 & 1.788 & 0.973 & 0.454 & 3.051 & 0.723 & 0.335 & 0.168 & 0.554 \\
\hline 200 & 30 & 80 & 1.881 & 1.133 & 0.442 & 3.485 & 0.786 & 0.399 & 0.168 & 0.578 \\
\hline
\end{tabular}

\begin{tabular}{c|c||c|c} 
Ordinal Series & Thresholds & Series & Thresholds \\
\hline 1 & $-1.5,-.75, .75,1.5$ & 2 & -.5 .5 \\
3 & 0 & 4 & -.50 .5 \\
5 & -.4 .41 & 6 & -101 \\
7 & .41 .6 & 8 & .3 \\
9 & 1 & 10 & 0.61 .2 \\
11 & .2 .6 & 12 & -.2 .6 \\
13 & -.70 .7 & 14 & -1.2 .21 .5 \\
15 & 0.751 .2 & & \\
\hline
\end{tabular}

For $y=X, x, G, Z$ where $Z$ denotes quantified data, $r_{y}^{2}$ denotes the number of factors estimated by the IC criterion of Bai and $\mathrm{Ng}(2002)$ with penalty $g_{2} . R_{y}^{2}$ is the average $R^{2}$ when each of the factors estimated from $y$ is regerssed on all the true factors. 
Table 6: Mixed Data, $r=2$

\begin{tabular}{cccc|cccc|cccc}
$N$ & $J_{\text {nominal }}$ & $J_{\text {ordinal }}$ & $n_{G}$ & $\widehat{r}_{X}$ & $\widehat{r}_{G}$ & $\widehat{r}_{Z}$ & $\widehat{r}_{x}$ & $R_{X}^{2}$ & $R_{G}^{2}$ & $R_{Z}^{2}$ & $R_{x}^{2}$ \\
\hline \multicolumn{1}{c}{} & & & & \multicolumn{9}{c}{$\theta=1$} \\
\hline 100 & 5 & 5 & 31 & 2.000 & 4.000 & 2.000 & 2.003 & 0.953 & 0.491 & 0.940 & 0.941 \\
100 & 10 & 5 & 42 & 2.000 & 4.000 & 2.000 & 2.002 & 0.957 & 0.497 & 0.942 & 0.943 \\
100 & 5 & 10 & 41 & 2.000 & 3.997 & 2.000 & 2.003 & 0.958 & 0.585 & 0.942 & 0.943 \\
200 & 5 & 5 & 31 & 2.000 & 4.000 & 2.000 & 2.003 & 0.953 & 0.470 & 0.942 & 0.941 \\
200 & 10 & 5 & 42 & 2.000 & 4.000 & 2.000 & 2.002 & 0.956 & 0.478 & 0.944 & 0.943 \\
200 & 5 & 10 & 41 & 2.000 & 4.000 & 2.000 & 2.004 & 0.958 & 0.542 & 0.944 & 0.943 \\
200 & 5 & 20 & 74 & 2.000 & 3.615 & 2.000 & 2.004 & 0.961 & 0.608 & 0.946 & 0.945 \\
200 & 10 & 20 & 85 & 2.000 & 3.467 & 2.000 & 2.003 & 0.963 & 0.646 & 0.948 & 0.947 \\
400 & 5 & 5 & 31 & 2.000 & 4.000 & 2.000 & 2.010 & 0.953 & 0.468 & 0.943 & 0.942 \\
400 & 10 & 5 & 42 & 2.000 & 4.000 & 2.000 & 2.007 & 0.957 & 0.471 & 0.945 & 0.944 \\
400 & 5 & 10 & 41 & 2.000 & 4.000 & 2.000 & 2.014 & 0.958 & 0.528 & 0.945 & 0.944 \\
400 & 5 & 20 & 74 & 2.000 & 3.716 & 2.000 & 2.012 & 0.961 & 0.595 & 0.947 & 0.946 \\
400 & 10 & 20 & 85 & 2.000 & 3.669 & 2.000 & 2.010 & 0.963 & 0.637 & 0.949 & 0.947 \\
\hline
\end{tabular}

\begin{tabular}{c|c||c|c} 
Nominal Series & Thresholds & Series & Thresholds \\
\hline 1 & $-1.5,-.75, .75,1.5$ & 2 & -.2 .51 \\
3 & 0.8 & 4 & -.50 .5 \\
5 & .41 & 6 & -101 \\
7 & .5 .5 & 8 & -.3 .3 \\
9 & -11 & 10 & -1.21 .2 \\
\hline
\end{tabular}

\begin{tabular}{c|c||c|c} 
Ordinal Series & Thresholds & Series & Thresholds \\
\hline 1 & .2 .6 & 2 & $-.2-.6$ \\
3 & -.70 .7 & 4 & -1.2 .21 .5 \\
5 & -.751 .2 & 6 & -1.3 \\
7 & -1 & 8 & -.7 \\
9 & 0 & 10 & .3 \\
11 & $-1.5,-.75, .75,1.5$ & 12 & -.5 .5 \\
13 & 0 & 14 & -.50 .5 \\
15 & -.4 .41 & 16 & -101 \\
17 & -.7 .41 .6 & 18 & -.3 \\
19 & 1 & 20 & -.61 .2 \\
\hline
\end{tabular}




\section{References}

Almund, M., A. Duckworth, J. Heckman, and T. Kautz (2011): "Personality Psychology and Economics," NBER working paper 16822.

Anderson, T. W., And H. Rubin (1956): "Statistical Inference in Factor Analysis," in Proceedings of the Third Berkeley Symposium on Mathematical Statistics and Probability, ed. by J. Neyman, vol. V, pp. 114-150. Berkeley: University of California Press.

Babakus, E., C. Ferguson, and K. Joreskog (1987): "The Sensitivity of Confirmatory Maximum Likelihood Factor Analysis to Violations of Measurement Scale and Distributonal Assumptions," Journal of Marketing Research, 37(720141).

BAI, J. (2003): "Inferential Theory for Factor Models of Large Dimensions," Econometrica, 71:1, $135-172$.

BAI, J., AND S. NG (2002): "Determining the Number of Factors in Approximate Factor Models," Econometrica, 70:1, 191-221.

\section{$3: 2,89-163$.}

(2008): "Large Dimensional Factor Analysis," Foundations and Trends in Econometrics,

Boivin, J., And S. NG (2006): "Are More Data Always Better for Factor Analysis," Journal of Econometrics, 132, 169-194.

Bollen, K. (1989): Structural Equations with Latent Variables. Wiley, New York.

Browne, M. W. (1984): "Asymptotically Distribution Free Methods in the Analysis of Covariance Structures," British Journal of Mathematical and Statistical Psychology, 37, 62-83.

BujA, A. (1990): "Remarks on Functional Canonical Variates: Alternating Least Squares Methods and ACE," Annals of Statistics, 18(3), 1032-1069.

Chamberlain, G., and M. Rothschild (1983): "Arbitrage, Factor Structure and Mean-Variance Analysis in Large Asset Markets," Econometrica, 51, 1281-2304.

Cliff, N. (1966): "Orthogonal Rotation to Congruence," Psychometrika, 31, 33-42.

Connor, G., And R. Korajzcyk (1986): "Performance Measurement with the Arbitrage Pricing Theory: A New Framework for Analysis," Journal of Financial Economics, 15, 373-394.

Conti, G., J. Heckman, H. Lopes, and R. Piatek (2012): "Constructing Economically Justified Aggregates: An Application to Early Orgins of Health," Journal of Econometrics.

Cunha, F., and J. Heckman (2008): "Formulating, Identifying and Estimating the Techology of Cognitive and Noncognitive Skill Formuation," Journal of Human Resources, 43(4), 738-782.

De Leeuw, J. (2004): "Least Squares Optimal Scaling of Partially Observed Linear Systems," in Recent Developments on Structural Equation Models, pp. 121-134. Kluwer Academic Publishers.

DE Leeuw, J. (2005): "Monotonic Regression," in Encyclopedia of Statistics in Behavioral Science, ed. by B. Everitt, and D. Howell, vol. 3, pp. 1260-1261. John Wiley and Sons.

Dolan, C. (1994): "Factor Analysis of Variables with 2,3,5, and 7 Response Categories: A Comparison of Categorical Variable Estimators Using Simulated Data," British Journal of Mathematical and Statistical Psychology, 46, 309-326.

Doz, C., D. Giannone, and L. Reichlin (2007): "A Quasi-Maximum Likelihood Approach for Large Approximate Dynamic Factor Models," ECARES working paper. 
Filmer, D., And L. Pritchett (1998): "Estimating Wealth Effect Without Expenditure Data - Or Tears: An Application to Educational Enrollments in States of India," Discussion Paper 1994, World Bank.

Gower, J., And G. Dijksterhuis (2004): Procrustes Problems. Oxford University Press.

Harman, H., and W. Jones (1966): "Factor Analysis by Minimizing Residuals (Minres)," Psychometrika, 31, 563-571.

IsRAels, A. (1984): "Redundancy Analysis for Qualitative Variables," Psychometrika, 49:3, 331346.

Joreskog, K. (1994): "On the Esetimation of Polychoric Correlations and their Asymptotic Covariance Matrix," Psychometrika, 59, 381-389.

Joreskog, K., And I. Moustki (2001): "Factor Analysis of Ordinal Variables: A Comparison of Three Approaches," Multivariate Behavioral Research, 36, 347-387.

Joreskog, K., and S. D. Sorbom (2006): LISREL User's Reference GuideChicago.

JoreskoG, K. G. (1970): "A Genearl Method for Analysis of Covariance Structurs," Biometrika, $57,239-51$.

(2003): "Factor Analysis by MINRES," www.ssicentral.com/lisrel/techdocs/minres.pdf.

Keller, W., and T. WAnsbeek (1983): "Multivariate Methods for Quantitative and Qualitative Data," Journal of Econometrics, 22, 91-111.

KIERs, H. (1990): "Majorization as a Tool for Optimizing a Class of Matrix Functions," Psyhometrika, 55(3), 417-428.

(2002): "Setting up Alternating Least Squares and Iterative Majorization Algorithms for Solving Various Matrix Optimization Problems," Computational Statistics and Data Analysis, $41,157-170$.

Kiers, H., Y. Takane, and A. MooijaArt (1993): "A Mononotically Convergent Algorithm for FACTALS," Psychometrika, 58(4), 567-574.

Kolenikov, S., And G. Angeles (2009): "Socioeconomic Status Measurement with Discrete Proxy Variables: Is Principal Component Analysis a Reliable Answer," Review of Income and Wealth, 55(128-165).

Kruskal, J. (1964): "Nonmetric Multidimensional Scaling: A Numerical Method," Psychometrika, 29, 115-129.

Kruskal, J., and R. Shepard (1974): "A Nonmetric Variety of Linear Factor Analysis," Psychometrika, 39, 123-157.

Lancaster, H. (1957): "Some Properties of the Bivariate Normal Distribution Considered in the form of a Contingency Table," Biometrika, 44:1/2, 289-292.

Lawley, D. N., And A. E. Maxwell (1971): Factor Analysis in a Statistical Method. Butterworth, London.

McDonald, R. P. (1985): Factor Analysis and Related Methods. Hilsdale, NJ: Erlbaum.

Meulman, J., And W. Heiser (2001): SPSS CategoriesSPSS Inc, 11.0 edn.

Michailidis, G., And J. De Leeuw (1998): "The Gifi System of Descriptive Multivariate Analysis," Statistical Science, 13(4), 307-336. 
Muthén, B. (1984): "A General Structural Equation Model with Dichotomous, Ordered, Categorical, and Continuous Latent Variable Indicators," Psychometrika, 49, 115-132.

Muthen, B., And D. Kaplan (1985): "A Comparison of Some Methodologies for the Factor Analysis of Non-normal Likert Variables," British Journal of Mathematical and Psychology, 38, $71-189$.

Nevels, K. (1989): "An Improved Solution for Factals: A Nonmetric Common Factor Analysis," Psychometrika, 54(3390343).

Olsson, U., F. Drasgow, and N. Dorans (1982): "The Polyserial Correlation Coefficient," Psychometrika, 47:3, 337-347.

Onatski, A. (2010): "Determining the Number of Factors From Empirical Distribution of Eigenvalues," Review of Economics and Statistics, 92:4, 1004-1016.

Pearson, K. (1900): "On the Correlation of Charcters not Quantitatively Measurable," in Mathematical Contributions to the Theory of Evolution: Philosophical Transactions of the Royal Society of London, Series A, vol. 195, pp. 1-46.

RACINE, J., AND Q. Li (2004): "Nonparametric Estimation of Regression Functions with Both Discrete and Continuous Data," Journal of Econometrics, 119, 99-130.

Schonmenn, P. (1966): "A Generalized Solution of the Orthogonal Procustes Problem," Psychometrika, 31(1), 1-10.

SocAn, G. (2003): "The Incremental Value of Minimum Rank Factor Analysis," Phd Thesis, University of Groningen.

Spearman, C. (1904): "General Intelligence, Objectively Determined and Measured," American Journal of Psychology, 15, 201-293.

Su, L., and A. Ullah (2009): "Functional Coefficient Estimation with Both Categorical and Continuous Data," in Advances in Econometrics, ed. by Q. Li, and J. Racine, vol. 25, pp. 131167. Emerald Group Publishing Limited.

Takane, Y., F. Young, and J. De Leeuw (1979): "Nonmetric Common Factor Analysis An Alternating Least Squares Method with Optimal Scaling Features," Behaviormetrika, 6, 45-56.

TEn Berge, J. (1993): Least Squares Optimization in Multivariate Analysis. DSWO Press, Leiden.

ten Berge, J., and K. Nevels (1997): "A General Solution to Mosier's Oblque Procrustes Problem," Psychometrika, 42, 593-600.

Tenenhaus, M., and F. Young (1985): "An Analysis and Synthesis of Multiple Correspondence Analysis, Ooptimal Scaling, Dual Scaling, Homogeneity Analysis and Other Methods for Quantifying Categorical Multivariate Data," Psychometrika, 50(1), 91-119.

Trendafilov, N., and S. Unkel (2011): "Exploratory Factor Analysis of Data Matrices with More Variables Than Observations," Journal of Computational Graphical Statistics, 20, forthcoming.

Unkel, S., And N. Trendafilov (2010): "Simultaneous Parameter Estimation in Exploratory Factor Analysis: An Expository Review," International Statistical Review, 78:3, 363-382. 